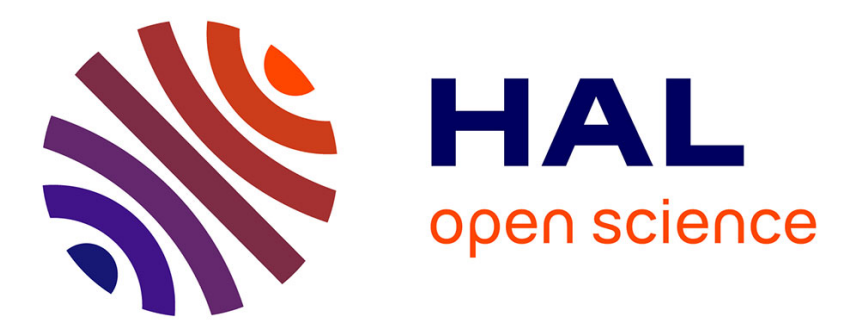

\title{
Multiscale model for flow and transport in CO2-enhanced coalbed methane recovery incorporating gas mixture adsorption effects
}

Tien Dung Le, Quoc Dat Ha, Irina Panfilov, Christian Moyne

\section{- To cite this version:}

Tien Dung Le, Quoc Dat Ha, Irina Panfilov, Christian Moyne. Multiscale model for flow and transport in CO2-enhanced coalbed methane recovery incorporating gas mixture adsorption effects. Advances in Water Resources, 2020, 144, pp.103706. 10.1016/j.advwatres.2020.103706 . hal-02922716

\section{HAL Id: hal-02922716 \\ https://hal.univ-lorraine.fr/hal-02922716}

Submitted on 26 Aug 2020

HAL is a multi-disciplinary open access archive for the deposit and dissemination of scientific research documents, whether they are published or not. The documents may come from teaching and research institutions in France or abroad, or from public or private research centers.
L'archive ouverte pluridisciplinaire HAL, est destinée au dépôt et à la diffusion de documents scientifiques de niveau recherche, publiés ou non, émanant des établissements d'enseignement et de recherche français ou étrangers, des laboratoires publics ou privés. 


\title{
Multiscale model for flow and transport in $\mathrm{CO}_{2}$-enhanced coalbed methane recovery incorporating gas mixture adsorption effects
}

\author{
T D Le ${ }^{\mathrm{a}, *}$, Q D Ha ${ }^{\mathrm{a}}$, I Panfilov ${ }^{\mathrm{a}}, \mathrm{C} \mathrm{Moyne}^{\mathrm{a}}$ \\ ${ }^{a}$ Université de Lorraine, CNRS, LEMTA, F-54000 Nancy, France
}

\begin{abstract}
In this work we develop a multiscale model for flow and transport problem in $\mathrm{CO}_{2}$-enhanced coalbed methane recovery. The coalbed methane reservoir is characterized by two levels of porosity associated with nanopores in the matrix and cleat network. Mass conservation equations for fluid mixture $\left(\mathrm{CH}_{4}\right.$ and $\left.\mathrm{CO}_{2}\right)$ in the matrix at the microscale are rigorously derived by using the formal homogenization technique taking into account the gas mixture adsorption in the nanopores. The Density Functional Theory (DFT) is used to compute the gas adsorption isotherms and the solvation force acting on the nanopore wall, showing a much more pronounced adsorption capacity of $\mathrm{CO}_{2}$ compared to $\mathrm{CH}_{4}$. The average transport equations in the matrix together with the multiphase flow problem in fracture network (gas mixture and water) are homogenized giving rise to a macroscopic model ruled by the effective conductivities, partition and transfer coefficients. The cleat permeability evolution due to deformation is taken into account through a three-scale poromechanical model reported in a previous work. Computational simulations illustrate the macroscopic laws underlying the gas pressure distributions, cleat closure phenomena and $\mathrm{CH}_{4}$ production curve enhanced by $\mathrm{CO}_{2}$ injection.

Keywords: Multiscale model, Dual porosity, Multiphase flow, Homogenization, Gas adsorption, Enhanced Coalbed Methane Recovery
\end{abstract}

\section{Introduction}

$\mathrm{CO}_{2}$-enhanced coalbed methane recovery consists of an unconventional gas resource and environmentally sustainable procedure which combines methane production with $\mathrm{CO}_{2}$ sequestration. In order to successfully apply this enhanced recovery procedure, one needs to describe accurately the coupled phenomena which 5 occur at disparate length scales. In this scenario, describing the anomalous behavior of the gas mixture in the nanopores and developing efficient upscaling procedure, capable of propagating the local description to obtain the macroscopic response, is a crucial issue. Coal seams are composed of a coal matrix containing

\footnotetext{
${ }^{*}$ Corresponding author

Email address: tien-dung.le@univ-lorraine.fr (T D Le )
} 
pores of several nanometers filled by adsorbed $\mathrm{CH}_{4}$ before exploration along with a fracture network (cleats) (see Fig. 1) with much higher permeability and partially saturated with water $[1,2,3,4]$. The classical primary gas exploration simply consists of decreasing the pore pressure leading to water production from the cleats and subsequently gas desorption from the matrix $[2,4]$. The enhanced coalbed methane recovery consists of a $\mathrm{CO}_{2}$ injection process which takes advantage of the preferential $\mathrm{CO}_{2}$ adsorption compared to $\mathrm{CH}_{4}[4,5,6]$. In spite of its advantage, this enhancement technique exhibits some drawbacks which stem from the matrix swelling inducing cleat closure and decrease in permeability [5, 7]. Such a mechanism is induced by the solvation force in the nanopores which is much more significant than the bulk pressure [8]. In this paper, we aim at clarifying this issue by developing a new multiscale model for flow and transport in dual porosity coalbed methane reservoir, which is coupled with the three-scale poromechanical model reported in a previous work [8].

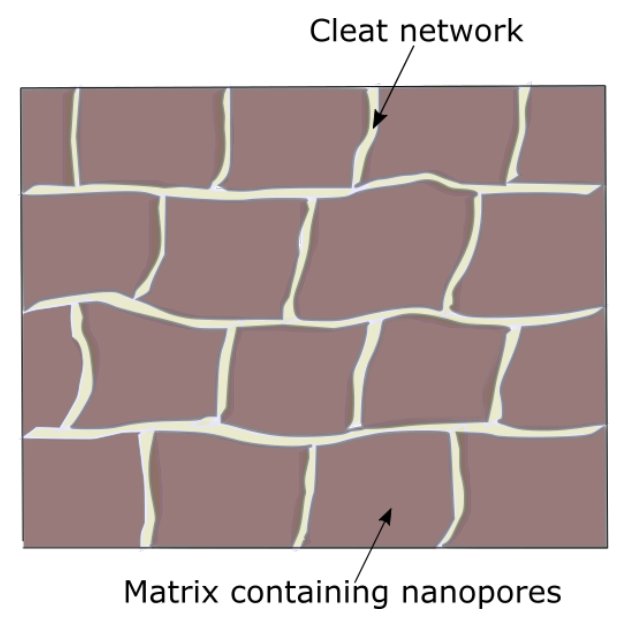

Figure 1: General structure of coalbed methane reservoir.

Modeling of the coupling between transport and poromechanics problems in coal seam is well reported in the literature and classically described by dual porosity type models combined with Langmuir isotherm to compute gas adsorption in the coal matrix $[9,10,11,12,13]$. Moreover, modeling of $\mathrm{CO}_{2}$-enhanced coalbed methane recovery has showed important improvement of methane production curve and matrix swelling phenomena inducing cleat closure $[10,11,12,14]$. These models have proposed empiric macroscopic models in which no closure for effective conductivities has been provided. Beyond the Langmuir approach, molecular dynamics and stochastic modeling have been explored in order to construct accurately the adsorption isotherms and study its influence on poromechanics $[8,15,16]$. Dual porosity models taking into account anomalous behavior of adsorbed fluid in the nanopores treated by statistical mechanics and its influence on the macroscopic constitutive laws through the homogenization method have been reported for applications to swelling clay and shale gas $[17,18,19,20]$. These approaches allow to construct precisely the macroscopic 

to the macroscale.

Despite the above-mentioned advances in coalbed methane modeling, a still open issue stems from the necessity of constructing a rigorous upscaling procedure capable of capturing the local anomalous behavior of the gas mixture in the nanopores of the coal matrix and propagating this information in order to derive Density Functional Theory (DFT) is applied to compute precisely the gas mixture adsorption quantities and the solvation force in the nanopores within the matrix. Gas transport in the matrix is ruled by Knudsen diffusion-type which is upscaled to the microscale in order to obtain the average mass conservation equations for gases in the matrix, characterized by the partition coefficients and effective diffusion coefficients. At the equations in the matrix. A second homogenization procedure is then applied to obtain a macroscopic reservoir-scale model for gas and water transport. The change in cleat porosity is taken into account by coupling this transport model with the three-scale poromechanical model reported in [8].

The paper is organized as follows. In Section 2, the three-scale poromechanics modeling developed by the

\subsection{Adsorption phenomena in nanopores}

The Density Functional Theory (DFT) is applied to describe precisely the gas mixture adsorption phenomena in nanopores $[8,21,22,23]$. The gas adsorption quantities and solvation force have been numerically computed for a spherical geometry by the authors [8]. Due to the gas confinement, the pressure exerted by the gas on the solid wall of the nanopores $P_{m}+\Pi$ is very different from the total bulk pressure in the matrix $P_{m}$. The solvation force $\Pi$ which is much larger than $P_{m}$ is the dominant force. In Fig. 2, the dependence of $\Pi$ on the partial bulk pressures (here $P_{\mathrm{CH}_{4}}^{b}$ and $P_{\mathrm{CO}_{2}}^{b}$ correspond to the partial bulk pressures in the 
matrix $P_{m, i}$ with $\left.i \in\left\{1=\mathrm{CH}_{4}, 2=\mathrm{CO}_{2}\right\}\right)$ is illustrated for a fixed pore diameter $D=5 d$ where $d$ is the molecule diameter (the both gas molecules are assumed to have the same diameter). We can observe that the absolute value of the solvation force is much higher than the bulk pressure. With a fixed $\mathrm{CH}_{4}$ pressure, the absolute value of the solvation force increases with the $\mathrm{CO}_{2}$ bulk pressure at small $\mathrm{CO}_{2}$ bulk pressure and then decreases at high $\mathrm{CO}_{2}$ bulk pressure. It should be noted that the solvation force reported in [8] does not keep the carbon molecule number at the solid surface constant. In this paper, the derivative of the external (wall) potential acting on the fluid molecules is made at fixed solid (carbon) molecule number. The solvation force is now negative. However, a similar result as the one obtained in [8] is that the derivative of the solvation force with respect to the $\mathrm{CO}_{2}$ bulk pressure is positive at high $\mathrm{CO}_{2}$ bulk pressure. This point is of utmost importance for the development of the poromechanics described in the following subsections.

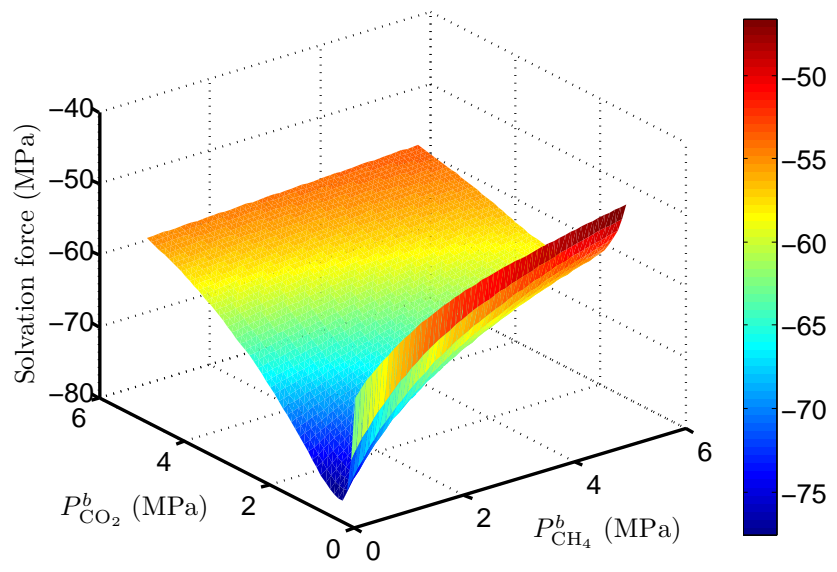

Figure 2: Dependence of the solvation force on the partial bulk pressures with fixed $D=5 d$

It was also discussed in [8] that the adsorption potential of $\mathrm{CO}_{2}$ is much more significant than $\mathrm{CH}_{4}$ so that $\mathrm{CO}_{2}$ injection can provide more methane from the matrix. This enhanced coalbed methane recovery technique will be discussed in details in Section 5 .

\subsection{Coal matrix}

The matrix is assumed poroelastic with an elasticity tensor $\mathbf{C}$ and a Biot-Willis coefficient $\alpha$. The effective stress principle for the matrix including the contribution from the solvation force reads as $[8,17,18]$

$$
\boldsymbol{\sigma}_{m}=\mathbf{C}: \mathcal{E}(\mathbf{u})-\alpha\left(P_{m}+\Pi\right) \boldsymbol{I} \quad \text { in } \Omega_{m}
$$

where $\boldsymbol{I}$ denotes the identity tensor and $\mathcal{E}(\mathbf{u})=\left(\boldsymbol{\nabla u}+(\boldsymbol{\nabla u})^{\mathrm{T}}\right) / 2$ the symmetric gradient of the deformation field $\mathbf{u}$. The fluid pressure acting on the wall of the nanopores is the sum of the bulk matrix pressure $P_{m}$ and the solvation force $\Pi$. For spherical pores, the disjoining stress reduces to a scalar component $\Pi$ which acts to expand the matrix and therefore to close the cleats. 
Linearizing the solvation force near a reference point leads to a new form of the effective stress principle for the matrix

$$
\boldsymbol{\sigma}_{m}-\boldsymbol{\sigma}_{m}^{r e f}=\mathbf{C}^{*}: \mathcal{E}\left(\mathbf{u}-\mathbf{u}^{r e f}\right)-\sum_{i} \alpha_{i}^{*}\left(P_{m, i}-P_{m, i}^{r e f}\right) \boldsymbol{I}
$$

with the modified poromechanical coefficients $\mathbf{C}^{*}$ and $\alpha_{i}^{*}$ given by

$$
\begin{aligned}
\mathbf{C}^{*} & =\mathbf{C}-\frac{\alpha^{2}}{1-\beta \frac{\partial \Pi}{\partial \phi_{m}}} \frac{\partial \Pi}{\partial \phi_{m}} \boldsymbol{I} \otimes \boldsymbol{I} \\
\alpha_{i}^{*} & =\frac{\alpha}{1-\beta \frac{\partial \Pi}{\partial \phi_{m}}}\left(1+\frac{\partial \Pi}{\partial P_{m, i}}\right)
\end{aligned}
$$

85 pressures $P_{m, i}$ in the reference configuration. Fig. 3 shows the dependence of the modified Biot-Willis

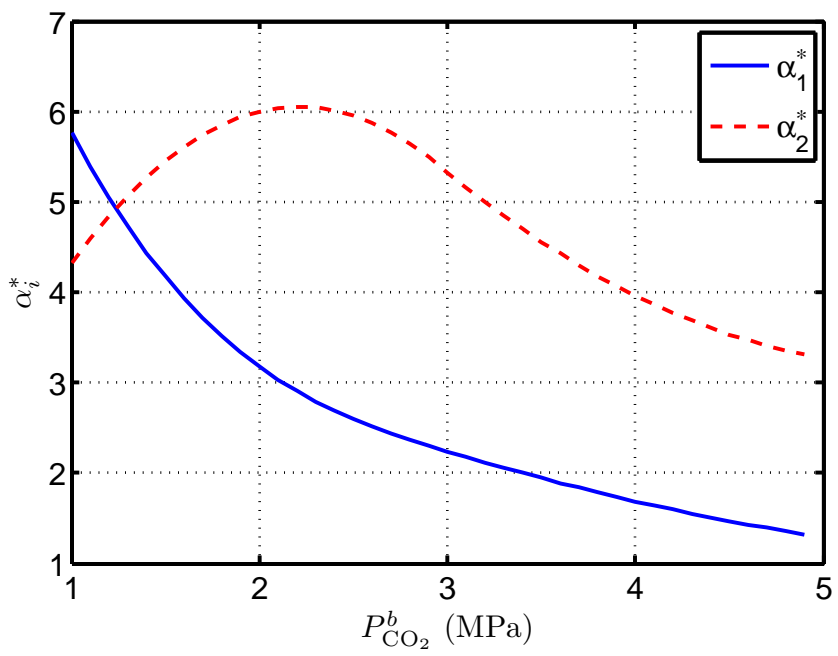

Figure 3: Dependence of $\alpha_{i}^{*}$ in $P_{\mathrm{CO}_{2}}^{b}$ for $P_{\mathrm{CH}_{4}}^{b}=1 \mathrm{MPa}$ and $D=5 d ; 1=\mathrm{CH}_{4} ; 2=\mathrm{CO}_{2}$.

parameters $\alpha_{i}^{*}$ for each gas component on the partial pressures. It is important to note that $\alpha_{i}^{*}$ can be higher than one in contrast to the classical Biot-Willis parameter, due to the solvation force effects.

\subsection{Macroscopic model}

The homogenization procedure of a three-scale poromechanics problem has been examined rigorously in [17] and applied to coalbed methane reservoir in [8]. The total stress tensor satisfies the equilibrium condition

$$
\nabla \cdot \sigma_{T}=0
$$

The displacement is the sum of three terms: an elastic contribution, a pressure component and a last contribution stemming from the solvation force. For the sake of simplicity, a quasi-steady version is considered 
under the assumption that the local equilibrium for each fluid component is quickly obtained between the displacement is neglected. The steady representations of the macroscopic stress tensor and the Lagrangian cleat porosity (i.e. the fluid volume relative to the initial total volume [24]) written in an incremental form read as [8]

$$
\begin{aligned}
\Delta \boldsymbol{\sigma}_{T} & =\overline{\mathbf{C}}: \mathcal{E}(\Delta \mathbf{u})+(\overline{\boldsymbol{\alpha}}-\boldsymbol{I}) \sum_{i}\left(\alpha_{i}^{*}-1\right) \Delta P_{f, i}-\Delta P_{f} \boldsymbol{I} \\
\Delta \bar{\phi}_{f} & =\overline{\boldsymbol{\alpha}}: \mathcal{E}(\Delta \mathbf{u})-\bar{\beta} \sum_{i}\left(\alpha_{i}^{*}-1\right) \Delta P_{f, i}
\end{aligned}
$$

where $\overline{\mathbf{C}}$ is the macroscopic elasticity tensor corresponding to a solid phase with an elastic tensor $\boldsymbol{C}^{*}$ and holes of porosity $\bar{\phi}_{f}$. For any variable $X, \Delta X=X(t+\Delta t)-X(t)$ where $\overline{\mathbf{C}}, \overline{\boldsymbol{\alpha}}, \bar{\beta}$ and $\alpha_{i}^{*}$ are constant over the time interval $\Delta t$ and then updated to compute the next time interval. In the isotropic case, the Biot's coefficients $\bar{\alpha}(\overline{\boldsymbol{\alpha}}=\bar{\alpha} \boldsymbol{I})$ and $\bar{\beta}$ are given by

$$
\begin{aligned}
\bar{\alpha} & =\left(1-\frac{\bar{K}}{K^{*}}\right) \\
\bar{\beta} & =\frac{\bar{\alpha}-\bar{\phi}_{f}}{K^{*}}
\end{aligned}
$$

where $K^{*}$ and $\bar{K}$ denote the bulk modulus of the matrix and of the three-scale matrix including the cleats respectively, corresponding to $\mathbf{C}^{*}$ and $\overline{\mathbf{C}}$. As $\bar{\beta}>0$, the most intriguing point in Eq. (6) is that when $\alpha_{i}^{*}>1$ (see Fig. 3), increasing the gas pressure in the cleats leads to a decrease of the cleats porosity $\bar{\phi}_{f}$ and to cleat closure.

\section{Mass conservation of a fluid mixture in the matrix}

We begin by constructing the average mass conservation equations for a gas mixture in the matrix. The matrix is composed of highly permeable disconnected inclusions (nanopores) locally in thermodynamic equilibrium with a virtual bulk and a solid phase occupying the sub-domains $\Omega_{n}$ and $\Omega_{s}$ respectively with an interface $\Gamma_{n s}$ (Fig. 4). The gas transport in the matrix is assumed to be a Knudsen diffusion mechanism in the solid phase whereas the nanopores play the role of gas storage sites. For a spherical geometry, the gas density profile within the nanopore was numerically computed by the authors [8] showing a highly inhomogeneous gas distribution.

Let $c_{s, i}$ be the gas concentration in the solid phase $\left(i \in\left\{\mathrm{CH}_{4}, \mathrm{CO}_{2}\right\}\right)$. By neglecting the storage capacity within the solid phase, the mass conservation equation reads as

$$
\boldsymbol{\nabla} \cdot\left(D_{s, i} \nabla c_{s, i}\right)=0 \quad \text { in } \Omega_{s}
$$

where $D_{s, i}$ denotes the diffusion coefficient in the solid phase. 


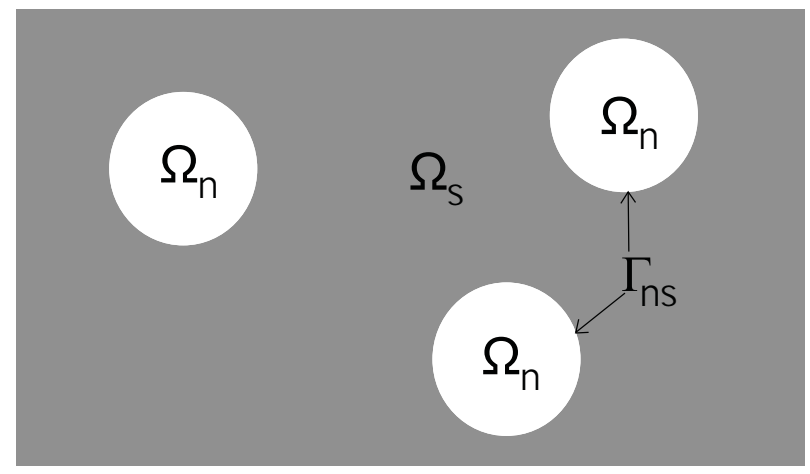

Figure 4: Structural configuration associated with nanopores and solid phase in coal matrix

In a consistent manner, the transport in the nanopores is assumed to be ruled by a diffusion mechanism in response to a virtual bulk concentration $c_{b, i}$. In addition, we will show later through the homogenization procedure that $c_{b, i}$ is nearly uniform in each pore. To describe the storage term in the nanopores, we introduce the partition coefficient $G_{i}$ so that $G_{i} c_{b, i}$ represents the gas quantity stored per unit volume in a nanopore in equilibrium with a bulk at concentration $c_{b, i}$. The transport in the nanopores is given as

$$
\frac{\partial\left(G_{i} c_{b, i}\right)}{\partial t}=\nabla \cdot\left(D_{b, i} \nabla c_{b, i}\right) \quad \text { in } \Omega_{n}
$$

where $D_{b, i}$ is the diffusion coefficient in the nanopores. It should be kept in mind that the nanopores are supposed to be in thermodynamic equilibrium with a virtual bulk so that the diffusion coefficient in the nanopores $D_{b, i}$ is assumed to be much larger than the diffusion coefficient in the solid phase $D_{s, i}$.

The accurate computation of $G_{i}$ can be achieved by the Density Functional Theory (DFT) [8]. Numerical results for $G_{i}$ will be presented in Section 5 .

In addition, the boundary conditions at the nanopores/solid phase interface $\Gamma_{n s}$ are given by

$$
\left\{\begin{aligned}
c_{b, i} & =c_{s, i} \\
-D_{n, i} \nabla c_{b, i} \cdot \boldsymbol{n} & =-D_{s, i} \boldsymbol{\nabla} c_{s, i} \cdot \boldsymbol{n}
\end{aligned}\right.
$$

The set of equations (9), (10) and (11) forms the pore-scale model for fluid transport in the matrix. Our aim now is to upscale this model in order to construct the average mass conservation equations for the matrix. To accomplish this task we proceed within the framework of the formal homogenization procedure with a spatially periodic structure for the matrix. Two length scales are introduced: a nanoscopic length $\left(l_{z}\right)$ of the size of the nanopores and a microscopic characteristic length $\left(l_{y}\right)$. The ratio between the two length scales $\varepsilon=l_{z} / l_{y}$ is the perturbation parameter assumed to be small. The perturbed models are now designated as $\varepsilon$-models. The periodic domain is reconstructed from a spatially repeated cell $Z^{\varepsilon}$, congruent to a standard cell $Z$, composed of the union of adjacent cell sub-domains $Z_{n}$ and $Z_{s}$ occupied by the nanopores and solid phase respectively with boundaries $\partial Z_{n s}$. The outer cell boundary, where the periodic condition 
is applied, is designated by $\partial Z_{s e}$. The problem consists in investigating the asymptotics of this problem as $\varepsilon \rightarrow 0$ and obtaining the homogenized limit as the scale of the heterogeneity tends to zero.

Within the current framework, introduce two coordinate systems: $\mathbf{y}$ at the microscale (slow coordinate) and $\mathbf{z}$ at the nanoscale (fast coordinate) together with its translation $\mathbf{z}=\mathbf{y} / \varepsilon$. The usual procedure consists level and collecting the successive powers of $\varepsilon$. Thus, postulate the ansatz

$$
f^{\varepsilon}(\mathbf{y}, \mathbf{z})=\sum_{k=0}^{\infty} \varepsilon^{k} f^{(k)}(\mathbf{y}, \mathbf{z})
$$

with the functions $f^{(i)}=f^{(i)}(\mathbf{y}, \mathbf{z})(i=0,1,2 \ldots)$, z-periodic. By adopting the macroscopic point of view in the sense of [25], the differential operator is replaced by

$$
\boldsymbol{\nabla} f^{\varepsilon}(\mathbf{y}, \mathbf{z})=\nabla_{y} f^{\varepsilon}(\mathbf{y}, \mathbf{z})+\varepsilon^{-1} \nabla_{z} f^{\varepsilon}(\mathbf{y}, \mathbf{z})
$$

To homogenize the preceding problem, the diffusion coefficient in the nanopores $D_{n, i}$ is supposed to be an order of magnitude larger $\left(\mathcal{O}\left(\epsilon^{-1}\right)\right)$ than the diffusion coefficient of the solid matrix $D_{s, i}[26,27]$. This assumption is based on the fact that the adsorbed gas in the nanopore is in thermodynamic equilibrium leading to an instantaneous diffusion whereas the gas transport mechanism within the solid phase is ruled by a Knudsen-type diffusion. After a scaling analysis, the problem at the pore-scale is written on a unit cell $Z=Z_{n} \bigcup Z_{s}$ as

$$
\left\{\begin{aligned}
\frac{\partial\left(G_{i} c_{b, i}\right)}{\partial t} & =\frac{1}{\epsilon} \boldsymbol{\nabla} \cdot\left(D_{n, i} \boldsymbol{\nabla} c_{b, i}\right) & & \text { in } Z_{n} \\
0 & =\boldsymbol{\nabla} \cdot\left(D_{s, i} \boldsymbol{\nabla} c_{s, i}\right) & & \text { in } Z_{s} \\
c_{b, i} & =c_{s, i} & & \text { on } \partial Z_{n s} \\
-\frac{1}{\epsilon} D_{n, i} \boldsymbol{\nabla} c_{b, i} \cdot \boldsymbol{n} & =-D_{s, i} \boldsymbol{\nabla} c_{s, i} \cdot \boldsymbol{n} & & \text { on } \partial Z_{n s}
\end{aligned}\right.
$$

From the above scaling analysis, the averaged transport equation in the matrix can be obtained by collecting the successive powers of $\epsilon$ (the details of this procedure are presented in Appendix B)

$$
\frac{\partial\left(\phi_{m} G_{i} c_{m, i}^{(0)}\right)}{\partial t}=\nabla_{y} \cdot\left(\boldsymbol{D}_{e q, i} \cdot \nabla_{y} c_{m, i}^{(0)}\right)
$$

with the effective diffusion coefficient defined as

$$
\boldsymbol{D}_{e q, i}=\frac{1}{|Z|}\left[\int_{\partial Z_{s e}} D_{s, i}\left(\mathbf{z} \otimes \boldsymbol{n}_{s}\right) \cdot\left(\boldsymbol{I}+\boldsymbol{\nabla}_{z} \boldsymbol{\chi}\right) \mathrm{d} S\right]
$$

where $\chi$ is the periodic vector in the unit cell satisfying the closure problem of $c_{s, i}^{(1)}$ in B.8.

It is important to note that this homogenization procedure is not classical since the diffusion coefficient 155 in the nanopores is an order of magnitude higher than the one in the solid phase. The homogenization result shows that the nanopores play the role of storage sites whereas the diffusion mechanism occurs only in the solid phase. 


\section{Macroscopic laws}

In what follows, the multiphase flow equations for gas and water in the cleats at the pore-scale will be constructed together with the preceding average diffusion equations of gas mixture in the matrix forming the microscopic model. This model will be upscaled to the macroscale to build-up the macroscopic constitutive laws at the reservoir scale.

\subsection{Microscopic model}

We consider the gas and water transport in the cleat system at the microscale. The cleat is filled by an immiscible mixture of gas and water phases. Let $\Omega_{m}$ and $\Omega_{f}$ be the volumes occupied by the matrix and the cleats. In addition, denote $\Gamma_{f m}$ the cleat/matrix interface. With assumption of incompressible water phase, transport of gas and water in the cleats is ruled by Darcy's law

$$
\begin{cases}\frac{\partial}{\partial t}\left[(1-S) \rho_{g, i}\right]-\nabla \cdot\left(\rho_{g, i} \frac{k_{f} k_{r g}}{\mu_{g}} \nabla P_{g}+D \rho_{g} \nabla \omega_{i}\right)=0 & \text { in } \Omega_{f} \\ \frac{\partial S}{\partial t}-\nabla \cdot\left(\frac{k_{f} k_{r w}}{\mu_{w}} \nabla P_{w}\right)=0 & \\ P_{g}=\sum_{i} P_{g, i} ; \quad \rho_{g}=\sum_{i} \rho_{g, i} ; \quad \omega_{i}=\rho_{g, i} / \rho_{g} & \end{cases}
$$

where $S$ and $\rho_{g, i}$ designate the water saturation and the gas density of component $i$ in the cleats. $P_{g}$ and $P_{w}$ are the total gas and water pressures whereas $k_{f}, k_{r g}$ and $k_{r w}$ are respectively the intrinsic permeability,

the gas and water relative permeabilities, $D$ the gas binary diffusion coefficient of $\mathrm{CO}_{2}$ and $\mathrm{CH}_{4}, \mu_{g}$ and $\mu_{w}$ the gas and water dynamic viscosity. The capillary pressure introduced as $P_{c}=P_{g}-P_{w}$ is only function of water saturation $S$. The gas and water fluxes are defined as

$$
\begin{aligned}
\boldsymbol{J}_{g, i} & =-\rho_{g, i} \frac{k_{f} k_{r g}}{\mu_{g}} \nabla P_{g}-D \rho_{g} \nabla \omega_{i} \\
\boldsymbol{J}_{w} & =-\frac{k_{f} k_{r w}}{\mu_{w}} \nabla P_{w}
\end{aligned}
$$

Omitting the superscript (0) and the subscript $y$ and rephrasing (15) in terms of mass flux, the mass conservation for gas transport in the matrix is rewritten as

$$
\frac{\partial\left(\phi_{m} G_{i} \rho_{m, i}\right)}{\partial t}+\nabla \cdot \boldsymbol{j}_{g, i}=0 \quad \text { in } \Omega_{m}
$$

where $\boldsymbol{j}_{g, i}=-\boldsymbol{D}_{e q, i} \boldsymbol{\nabla} \rho_{m, i}, \rho_{m, i}=M_{g, i} c_{m, i}$ and $M_{g, i}$ are respectively the gas flux, the mass density and the molar mass of species $i$ in the matrix.

The boundary condition at the cleat/matrix interface $\Gamma_{f m}$ is given by the continuity of flux and pressure

$$
\left\{\begin{array}{rlr}
\boldsymbol{J}_{g, i} \cdot \boldsymbol{n}_{f m} & =\boldsymbol{j}_{g, i} \cdot \boldsymbol{n}_{f m} \\
P_{m, i} & =P_{g, i}
\end{array} \quad \text { on } \Gamma_{f m}\right.
$$

where $P_{m, i}$ denotes the partial bulk matrix pressure of component $i$ constructed from the equation of state with given $\rho_{m, i}$. Here the pressure is assumed to be continuous at the interface rather than the concentration 
used in the previous section in order to simplify the upscaling of the Darcy equations in the cleats in which

the pressure is used.

\subsection{Upscaling to macroscale}

In the same way as the homogenization procedure used in Section 3 for the matrix, we now upscale the preceding microscopic model to obtain the macroscopic constitutive laws for transport in the coal reservoir.

The periodic cell $Y^{\varepsilon}$ is given by the union of adjacent cell sub-domains $Y_{m}^{\varepsilon}$ and $Y_{f}^{\varepsilon}$ occupied by matrix 185

and cleat respectively. Each cell is congruent to a standard unitary cell $Y$ composed of sub-domains $Y_{m}$ and $Y_{f}$ with boundaries $\partial Y_{f m}$. The two coordinates $\mathbf{x}$ and $\mathbf{y}$ represent now micro and macroscale (or fast and slow coordinates respectively) so that we have $\mathbf{y}=\mathbf{x} / \varepsilon$. We postulate the ansatz similar to (12) with the same derivation rule as (13).

The $\varepsilon$-microscopic model for the transport problem in the cleats reads as

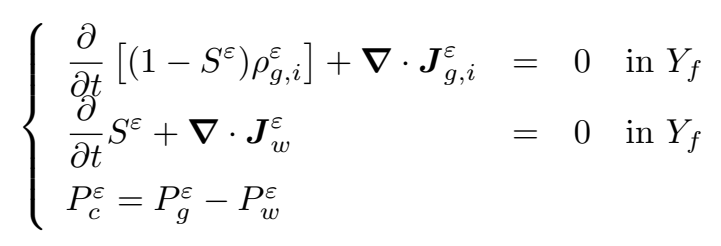

with

$$
\begin{aligned}
\boldsymbol{J}_{g, i}^{\varepsilon} & =-\rho_{g, i}^{\varepsilon} \frac{k_{f} k_{r g}}{\mu_{g}} \nabla P_{g}^{\varepsilon}-D \rho_{g}^{\varepsilon} \nabla \omega_{i}^{\varepsilon} \\
\boldsymbol{J}_{w}^{\varepsilon} & =-\frac{k_{f} k_{r w}}{\mu_{w}} \nabla P_{g}^{\varepsilon}+\frac{k_{f} k_{r w}}{\mu_{w}} \nabla P_{c}^{\varepsilon}
\end{aligned}
$$

190

The $\varepsilon$-model for gas transport in the matrix is given by

$$
\frac{\partial\left(\phi_{m} G_{i}^{\varepsilon} \rho_{m, i}^{\varepsilon}\right)}{\partial t}+\nabla \cdot j_{g, i}^{\varepsilon}=0 \quad \text { in } Y_{m}
$$

In the matrix, gas diffusion mechanism is a Knudsen diffusion-type while a molecular diffusion is considered in the cleats. Therefore, the diffusion coefficient in the matrix $\boldsymbol{D}_{e q, i}$ is much lower than the one in the cleats $D$, as shown in Table 1 . In the context of dual porosity model and based on an order of magnitude estimate, it is relevant to consider that $\boldsymbol{D}_{e q, i}=\varepsilon^{2} D$. Consequently, the first non-zero order of $\boldsymbol{j}_{g, i}^{\varepsilon}$ is $\boldsymbol{j}_{g, i}^{(1)}$ so that we ${ }_{195}$ have $\boldsymbol{j}_{g, i}^{\varepsilon}=-\varepsilon^{2} \boldsymbol{D}_{e q, i} \cdot \nabla \rho_{m, i}^{\varepsilon}[17,18,19]$.

The boundary conditions read as

$$
\left\{\begin{array}{rlr}
\boldsymbol{J}_{g, i}^{\varepsilon} \cdot \boldsymbol{n}_{f m} & =\boldsymbol{j}_{g, i}^{\varepsilon} \cdot \boldsymbol{n}_{f m} \\
P_{m, i}^{\varepsilon} & =P_{f, i}^{\varepsilon}
\end{array} \quad \text { on } \partial Y_{f m}\right.
$$

We now collect the successive powers of $\varepsilon$ in the above system. 


\section{Slow variables}

By invoking (21) at the order $\mathcal{O}\left(\varepsilon^{-2}\right)$ and the order $(24)$ at $\mathcal{O}\left(\varepsilon^{-1}\right)$ we obtain

$$
\left\{\begin{array}{l}
\boldsymbol{\nabla}_{y} \cdot\left(\rho_{g, i}^{(0)} \frac{k_{f} k_{r g}}{\mu_{g}} \nabla_{y} P_{g}^{(0)}+D \rho_{g}^{(0)} \nabla_{y} \omega_{i}^{(0)}\right) \\
-\nabla_{y} \cdot\left(\frac{k_{f} k_{r w}}{\mu_{w}} \nabla_{y} P_{g}^{(0)}\right)+\nabla_{y} \cdot\left(\frac{k_{f} k_{r w}}{\mu_{w}} \nabla_{y} P_{c}^{(0)}\right)=0
\end{array} \text { in } Y_{f}\right.
$$

and

$$
\left(\rho_{g, i}^{(0)} \frac{k_{f} k_{r g}}{\mu_{g}} \nabla_{y} P_{g}^{(0)}+D \rho_{g}^{(0)} \nabla_{y} \omega_{i}^{(0)}\right) \cdot \boldsymbol{n}=0 \quad \text { on } \Gamma_{f m}
$$

The non-oscillating variables are therefore

$$
P_{g, i}^{(0)}(\mathbf{x}, \mathbf{y}, t)=P_{g, i}^{(0)}(\mathbf{x}, t) \quad \omega_{i}^{(0)}(\mathbf{x}, \mathbf{y}, t)=\omega_{i}^{(0)}(\mathbf{x}, t) \quad P_{c}^{(0)}(\mathbf{x}, \mathbf{y}, t)=P_{c}^{(0)}(\mathbf{x}, t)
$$

In addition, $P_{c}^{(0)}$ is nothing else than a saturation dependent variable so that $S^{(0)}(\mathbf{x}, \mathbf{y}, t)=S^{(0)}(\mathbf{x}, t)$.

\section{Fluctuation terms}

By invoking equation (21) at the order $\mathcal{O}\left(\varepsilon^{-1}\right)$ and equation $(24)$ at the order $\mathcal{O}\left(\varepsilon^{0}\right)$ we obtain

$$
\left\{\begin{array}{r}
\boldsymbol{\nabla}_{y} \cdot\left[\rho_{g, i}^{(0)} \frac{k_{f} k_{r g}}{\mu_{g}} \nabla_{y} P_{g}^{(1)}+D \rho_{g}^{(0)} \nabla_{y} \omega_{i}^{(1)}\right]=0 \\
-\nabla_{y} \cdot\left[\frac{k_{f} k_{r w}}{\mu_{w}} \nabla_{y} P_{g}^{(1)}\right]+\nabla_{y} \cdot\left[\frac{k_{f} k_{r w}}{\mu_{w}} \nabla_{y} P_{c}^{(1)}\right]=0
\end{array} \text { in } Y_{f}\right.
$$

and

$$
\left\{\begin{array}{l}
{\left[\rho_{g, i}^{(0)} \frac{k_{f} k_{r g}}{\mu_{g}}\left(\nabla_{x} P_{g}^{(0)}+\nabla_{y} P_{g}^{(1)}\right)+D \rho_{g}^{(0)}\left(\nabla_{x} \omega_{i}^{(0)}+\nabla_{y} \omega_{i}^{(1)}\right)\right] \cdot \boldsymbol{n}=0} \\
{\left[-\frac{k_{f} k_{r g}}{\mu_{g}}\left(\nabla_{x} P_{g}^{(0)}+\nabla_{y} P_{g}^{(1)}\right)+\frac{k_{f} k_{r w}}{\mu_{w}}\left(\nabla_{x} P_{c}^{(0)}+\nabla_{y} P_{c}^{(1)}\right)\right] \cdot \boldsymbol{n}=0}
\end{array} \text { on } \Gamma_{f m}\right.
$$

By definition $\sum_{i} \omega_{i}=1$, hence we obtain $\sum_{i} \omega_{i}^{(1)}=0$. By summing (28a) and (29a) with respect to $i$ together with $(29 \mathrm{~b})$ we obtain

$$
\left\{\begin{aligned}
\nabla_{y} \cdot\left(\rho_{g}^{(0)} \frac{k_{f} k_{r g}}{\mu_{g}} \nabla_{y} P_{g}^{(1)}\right) & =0 \\
\nabla_{y} \cdot\left(\rho_{g}^{(0)} D \nabla_{y} \omega_{i}^{(1)}\right) & =0
\end{aligned} \quad \text { in } Y_{f}\right.
$$

and

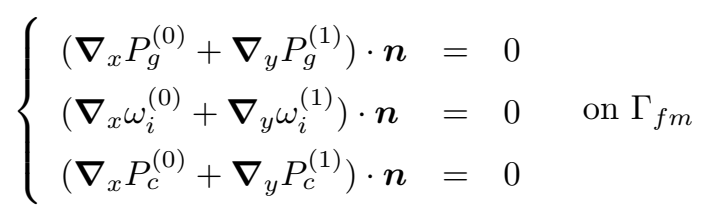

By invoking linearity and periodicity on the outer boundary of the unit cell, the classical closures for $P_{g}^{(1)}$, $\omega_{i}^{(1)}$ and $P_{c}^{(1)}$ are given by

$$
\begin{aligned}
P_{g}^{(1)} & =\boldsymbol{\varsigma}(\mathbf{y}) \cdot \nabla_{x} P_{g}^{(0)}+\widehat{x}_{g}(\mathbf{x}) \\
\omega_{i}^{(1)} & =\boldsymbol{\varsigma}(\mathbf{y}) \cdot \nabla_{x} \omega_{i}^{(0)}+\widehat{\omega}_{i}(\mathbf{x}) \\
P_{c}^{(1)} & =\boldsymbol{\varsigma}(\mathbf{y}) \cdot \nabla_{x} P_{c}^{(0)}+\widehat{x}_{c}(\mathbf{x})
\end{aligned}
$$


where $\varsigma$ Y-periodic satisfies

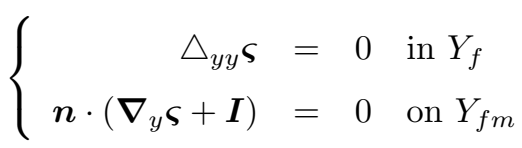

\section{Averaged equations} invoking (21) and (23) at the order $\mathcal{O}\left(\varepsilon^{0}\right)$ we obtain

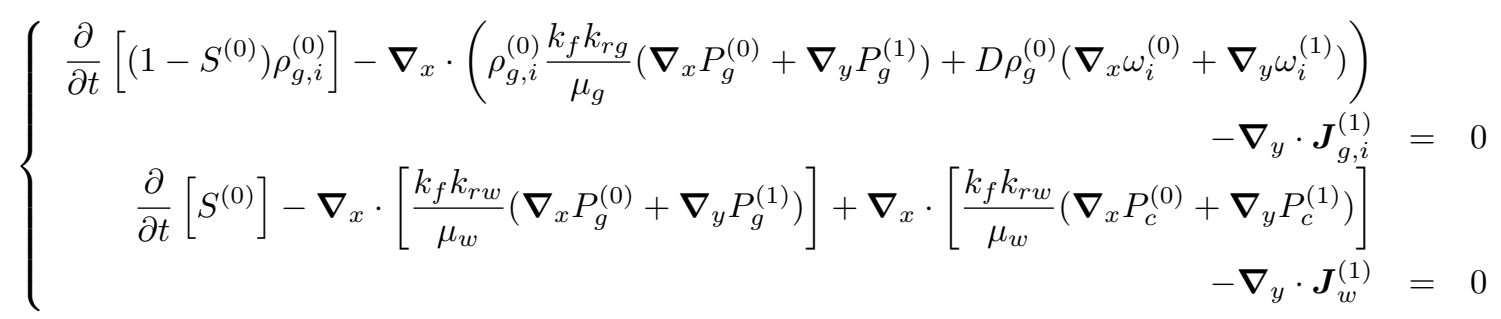

and

$$
\frac{\partial\left(\phi_{m} G_{i}^{(0)} \rho_{m, i}^{(0)}\right)}{\partial t}+\nabla_{y} \cdot \boldsymbol{j}_{g, i}^{(1)}=0
$$

with $\boldsymbol{j}_{g, i}^{(1)}=-\boldsymbol{D}_{e q, i} \cdot \nabla_{y} \rho_{m, i}^{(0)}$. The boundary conditions are given by

$$
\left\{\begin{aligned}
\rho_{m, i}^{(1)} & =\rho_{g, i}^{(1)} \\
\boldsymbol{J}_{g, i}^{(1)} \cdot \boldsymbol{n}_{f m} & =\boldsymbol{j}_{g, i}^{(1)} \cdot \boldsymbol{n}_{f m} \quad \text { on } \partial Y_{f m} \\
\boldsymbol{J}_{w}^{(1)} \cdot \boldsymbol{n}_{f m} & =0
\end{aligned}\right.
$$

By averaging mass conservation (34) on the unit cell combined with boundary condition (36), the transport equation for the species $i$ of the gas phase is written as

$$
\begin{aligned}
\frac{\partial}{\partial t}\left[\bar{\phi}_{f}\left(1-S^{(0)}\right) \rho_{g, i}^{(0)}\right]-\boldsymbol{\nabla}_{x} \cdot\left(\rho_{g, i}^{(0)} \frac{k_{f} k_{r g}}{\mu_{g}}\langle\boldsymbol{\nabla} \boldsymbol{\varsigma}\right. & \left.+\boldsymbol{I}\rangle \cdot \boldsymbol{\nabla}_{x} P_{g}^{(0)}+\rho_{g}^{(0)} D\langle\boldsymbol{\nabla} \boldsymbol{\varsigma}+\boldsymbol{I}\rangle \cdot \boldsymbol{\nabla}_{x} \omega_{i}^{(0)}\right)=-\left\langle\boldsymbol{\nabla}_{y} \cdot \boldsymbol{J}_{g, i}^{(1)}\right\rangle \\
=-\frac{1}{|Y|} \int_{\partial Y_{f m}} \boldsymbol{J}_{g, i}^{(1)} \cdot \boldsymbol{n}_{f m} \mathrm{~d} \Gamma & =\frac{1}{|Y|} \int_{\partial Y_{f m}} \boldsymbol{j}_{g, i}^{(1)} \cdot \boldsymbol{n}_{m f} \mathrm{~d} \Gamma=\left\langle\boldsymbol{\nabla}_{y} \cdot \boldsymbol{j}_{g, i}^{(1)}\right\rangle \\
& =-\frac{\partial\left\langle\phi_{m} G_{i}^{(0)} \rho_{m, i}^{(0)}\right\rangle}{\partial t}=-\frac{\partial\left[\left(1-\bar{\phi}_{f}\right) \phi_{m} G_{i}^{(0)}\left\langle\rho_{m, i}^{(0)}\right\rangle^{m}\right]}{\partial t}
\end{aligned}
$$

and for the water phase inside the cleats

$$
\frac{\partial}{\partial t}\left[\bar{\phi}_{f} S^{(0)}\right]-\boldsymbol{\nabla}_{x} \cdot\left(\frac{k_{f} k_{r w}}{\mu_{w}}\langle\nabla \boldsymbol{\varsigma}+\boldsymbol{I}\rangle \cdot \nabla_{x} P_{g}^{(0)}\right)+\boldsymbol{\nabla}_{x} \cdot\left(\frac{k_{f} k_{r w}}{\mu_{w}}\langle\boldsymbol{\nabla} \boldsymbol{\varsigma}+\boldsymbol{I}\rangle \cdot \boldsymbol{\nabla}_{x} P_{c}^{(0)}\right)=0
$$

In the context of dual porosity model, the cell problem for the mass transport inside the matrix given by (15) with the Dirichlet boundary condition, $\rho_{m}^{(0)}=\rho_{f}^{(0)}$ on $\partial Y_{f m}$ has to be solved at each point. A good approximation can be obtained by replacing this source term by a constant mass transfer coefficient between the matrix and the cleats (see Appendix A)

$$
\frac{\partial\left[\left(1-\bar{\phi}_{f}\right) \phi_{m} G_{i}^{(0)}\left\langle\rho_{m, i}^{(0)}\right\rangle^{m}\right]}{\partial t}=\alpha_{t, i}\left(\rho_{g, i}^{(0)}-\left\langle\rho_{m, i}^{(0)}\right\rangle^{m}\right)
$$


where $\alpha_{t, i}$ represents a transfer coefficient depending on the local geometry. The gas mass conservation in the cleats is finally given by

$$
\frac{\partial}{\partial t}\left[\bar{\phi}_{f}\left(1-S^{(0)}\right) \rho_{g, i}^{(0)}\right]-\nabla_{x} \cdot\left(\rho_{g, i}^{(0)} \boldsymbol{K}^{e} \cdot \nabla_{x} P_{g}^{(0)}+\rho_{g}^{(0)} \boldsymbol{D}^{e} \cdot \nabla_{x} \omega_{i}^{(0)}\right)=\alpha_{t, i}\left(\left\langle\rho_{m, i}^{(0)}\right\rangle^{m}-\rho_{g, i}^{(0)}\right)
$$

${ }_{210}$ with macroscopic effective coefficients $\boldsymbol{K}^{e}=\frac{K_{f} k_{r g}}{\mu_{g}}$ and $\boldsymbol{D}^{e}=D\langle\nabla \boldsymbol{\nabla}+\boldsymbol{I}\rangle$ where $K_{f}=k_{f}\langle\nabla \boldsymbol{\nabla}+\boldsymbol{I}\rangle$ is the apparent cleat permeability.

\section{Numerical simulation results}

In this section, numerical simulations are carried out by solving the macroscopic equations in COMSOL Multiphysics software for a 2D-coalbed reservoir geometry of dimension $150 \mathrm{~m} \times 150 \mathrm{~m}$ as given in Fig. 5 . $\mathrm{CO}_{2}$ can be injected in the injection well at the lower left corner of the reservoir in order to enhance $\mathrm{CH}_{4}$ production. We assume that the $\mathrm{CO}_{2}$ injection proceeds after the water depletion procedure, therefore the water saturation is taken as constant and close to the water residual saturation value. In addition, it should be noted that the multiscale model can also be used to simulate the water depletion phase by solving Eq. (38). The parameters used in the simulations are given in Table 1.

We consider a particular configuration in which the initial conditions satisfy $\alpha_{i}^{*}>1$ in order to observe the cleat closure phenomena. For initial conditions, the $\mathrm{CH}_{4}$ and $\mathrm{CO}_{2}$ pressures in the reservoir are uniform at $2 \mathrm{MPa}$ and $2 \mathrm{MPa}$ respectively. At the edges of the reservoir, no flux condition is imposed and the displacement is taken to be zero, i.e. the overall reservoir volume is constant. At the injection well, $\mathrm{CO}_{2}$ is injected at $5 \mathrm{MPa}$ whereas zero methane flux is applied. At the production well, Dirichlet boundary conditions for $\mathrm{CH}_{4}$ and $\mathrm{CO}_{2}$ pressures are taken at $0.1 \mathrm{MPa}$ and $2 \mathrm{MPa}$ respectively.

The transfer coefficients $\alpha_{t, i}$ are given by [28]

$$
\alpha_{t, i}=\frac{2 \pi^{2}}{s^{2}} D_{e q, i}
$$

where $s$ is the spacing of cleats. The characteristic diffusion time in the matrix can be estimated from Eq. 39 as

$$
t_{d}=\frac{\left(1-\bar{\phi}_{f}\right) \phi_{m} G_{i}}{\alpha_{t, i}}
$$

For $D_{e q, 1}=10^{-11} \mathrm{~m}^{2} \cdot \mathrm{s}^{-1}$ and $D_{e q, 2}=6.10^{-12} \mathrm{~m}^{2} \cdot \mathrm{s}^{-1}$, the characteristic time for the two gases is several days so that very small pressure differences are observed between the matrix and the cleats.

Firstly, in Fig. 6, the partition coefficients $G_{1}$ and $G_{2}$ relative to $\mathrm{CH}_{4}$ and $\mathrm{CO}_{2}$ depending on the partial pressures are computed by using the DFT for a spherical geometry. We may observe that the partition coefficient values for $\mathrm{CO}_{2}\left(G_{2}\right)$ are much higher than the those for $\mathrm{CH}_{4}\left(G_{1}\right)$ showing an adsorption potential 
of $\mathrm{CO}_{2}$ significantly higher compared to $\mathrm{CH}_{4}$. Moreover, the partition coefficients decrease with the increase in partial pressures.

In Fig. 7, the methane pressure distributions in the cleats for four different times $(1,12,24$ and 36 months) are displayed. We can observe the depletion with time of the methane pressure and a much more pronounced decrease near the production well. Due to $\mathrm{CO}_{2}$ injection, $\mathrm{CH}_{4}$ is replaced by $\mathrm{CO}_{2}$ leading to a significant pressure decrease near the injection well.

Fig. 8 depicts the $\mathrm{CO}_{2}$ pressure distributions in the reservoir for four different times. The $\mathrm{CO}_{2}$ pressure increases in the vicinity of the production well whereas the injected $\mathrm{CO}_{2}$ does not yet reach the production well after 36 months of injection.

The cleat porosity change is taken into account by coupling the transport model with the three-scale poromechanical model. The cleat permeability is related to the cleat porosity by the cubic relationship

$$
\frac{K_{f}}{K_{f}^{0}}=\left(\frac{\bar{\phi}_{f}}{\bar{\phi}_{f}^{0}}\right)^{3}
$$

The cleat porosity for different times is represented in Fig. 9 highlighting an important decrease in porosity near the injection well and this decrease propagates to the production well during the injection period. On the other hand, near the production well, the total pressure decrease leads to a matrix shrinkage and to an increase of the cleat porosity. Moreover, the ratio between the cleat permeability and its initial value is illustrated in Fig. 10. Near the injection well, the cleat permeability decreases significantly due to a strong matrix swelling induced by $\mathrm{CO}_{2}$ adsorption. As observed at the nanoscale by the DFT approach, the adsorption potential of $\mathrm{CO}_{2}$ is higher than $\mathrm{CH}_{4}$. Therefore $\mathrm{CO}_{2}$ can replace $\mathrm{CH}_{4}$ in the nanopores inducing a less attractive solvation force which swells the matrix. Particular attention must be paid to the injection pressure to avoid closing totally the cleats near the injection well.

Finally, the methane production curves are plotted in Fig. 11 for both cases "without" and "with" injection. The numerical results show a very promising technique of enhanced coalbed methane recovery. In this configuration, the injection of $\mathrm{CO}_{2}$ allows $16 \%$ more methane to be recovered than in the scenario without injection.

\section{Conclusion}

In this work a three-scale model for flow and transport in coalbed methane reservoir enhanced by $\mathrm{CO}_{2}$ injection has been developed. The coalbed methane reservoir is characterized by a matrix composed of solid and nanopores saturated by adsorbed gas and a cleat network. The DFT approach allows to compute precisely the gas mixture quantities adsorbed in the matrix and the solvation force acting on the nanopores surface. A first homogenization procedure was used to obtain the average mass conservation equations for the two gases $\left(\mathrm{CH}_{4}\right.$ and $\left.\mathrm{CO}_{2}\right)$ in the matrix taking into account the adsorption phenomena characterized 


\begin{tabular}{|c|c|c|c|}
\hline Parameter & Symbol & Value & Unit \\
\hline Ideal gas constant & $R$ & 8.314 & J.mol ${ }^{-1} \cdot \mathrm{K}^{-1}$ \\
\hline Temperature & $T$ & 310 & $\mathrm{~K}$ \\
\hline Mass molar of $\mathrm{CH}_{4}$ & $M_{1}$ & 16 & g.mol ${ }^{-1}$ \\
\hline Mass molar of $\mathrm{CO}_{2}$ & $M_{2}$ & 44 & g.mol ${ }^{-1}$ \\
\hline Gas viscosity & $\mu_{g}$ & $1.210^{-5}$ & Pa.s \\
\hline Gas residual saturation & $S_{r g}$ & 0.1 & - \\
\hline Water residual saturation & $S_{r w}$ & 0.15 & - \\
\hline Initial cleat permeability & $K_{f}^{0}$ & 5. $10^{-16}$ & $\mathrm{~m}^{2}$ \\
\hline Diffusion coefficient in matrix of $\mathrm{CH}_{4}$ & $D_{e q, 1}$ & $10^{-11}$ & $\mathrm{~m}^{2} \cdot \mathrm{s}^{-1}$ \\
\hline Diffusion coefficient in matrix of $\mathrm{CO}_{2}$ & $D_{e q, 2}$ & 6. $10^{-12}$ & $\mathrm{~m}^{2} \cdot \mathrm{s}^{-1}$ \\
\hline Gas binary diffusion coefficient & $D$ & 5. $10^{-5}$ & $\mathrm{~m}^{2} \cdot \mathrm{s}^{-1}$ \\
\hline Spacing of cleats & $s$ & 0.01 & $\mathrm{~m}$ \\
\hline Initial cleat porosity & $\bar{\phi}_{f}$ & 0.005 & - \\
\hline Matrix porosity & $\phi_{m}$ & 0.03 & - \\
\hline Matrix Biot's coefficient & $\alpha$ & 0.9 & - \\
\hline Young modulus & $E$ & 4.5 & $\mathrm{GPa}$ \\
\hline Poisson's ratio & $\nu$ & 0.3 & - \\
\hline Initial pressure of $\mathrm{CH}_{4}$ & $P_{1}^{i n i}$ & 2 & $\mathrm{MPa}$ \\
\hline Initial pressure of $\mathrm{CO}_{2}$ & $P_{2}^{i n i}$ & 2 & $\mathrm{MPa}$ \\
\hline Water saturation & $S$ & 0.2 & - \\
\hline
\end{tabular}

Table 1: Parameters used in the simulations 


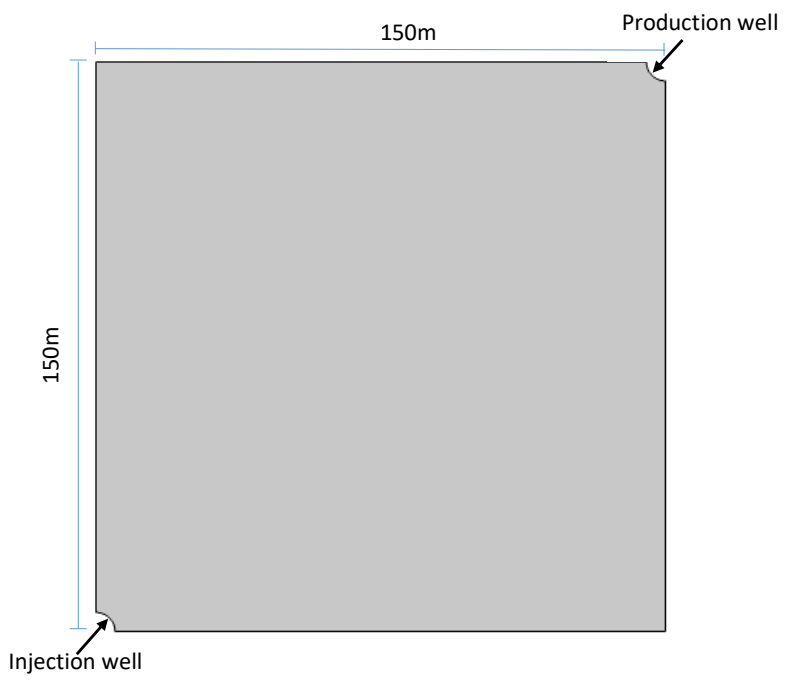

Figure 5: Computational domain
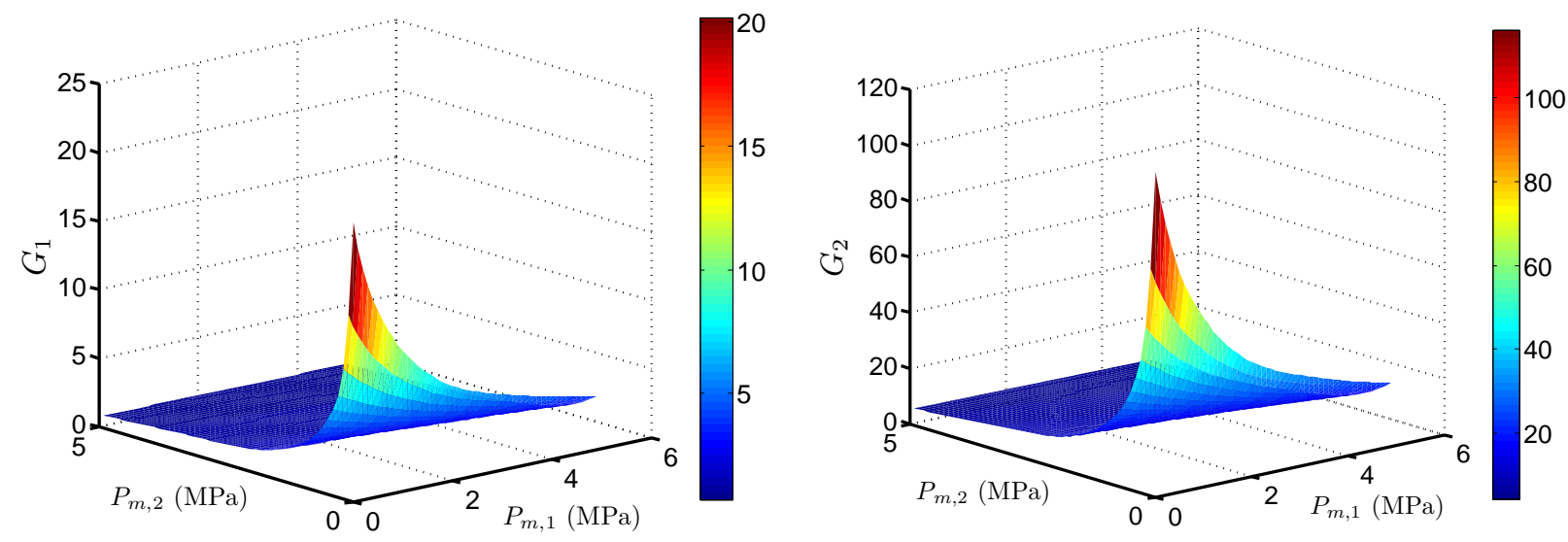

Figure 6: Dependence of the partition coefficients on the partial pressures

by a partition coefficient and an effective Knudsen-type diffusion. Special attention was paid to upscale the problem of disconnected highly permeable nanopores immersed in a low permeable matrix incorporating adsorption phenomena effects. A second homogenization procedure was applied to obtain macroscopic averaged equations for gas diffusion in the matrix and gas/water transport in the cleats. A dual porosity model of Warren and Root-type approximates the mass exchange between matrix and cleats through a matrix-fracture transfer shape factor.

The current model for flow and transport in coalbed methane reservoir is coupled with the three-scale poromechanical model developed in a previous work to construct a full model which accurately describes the simultaneous mechanics and transport multiscale problems in a coalbed methane reservoir. With $\mathrm{CO}_{2}$ injection such a model is capable of explaining the improvement of the methane production curve and also 

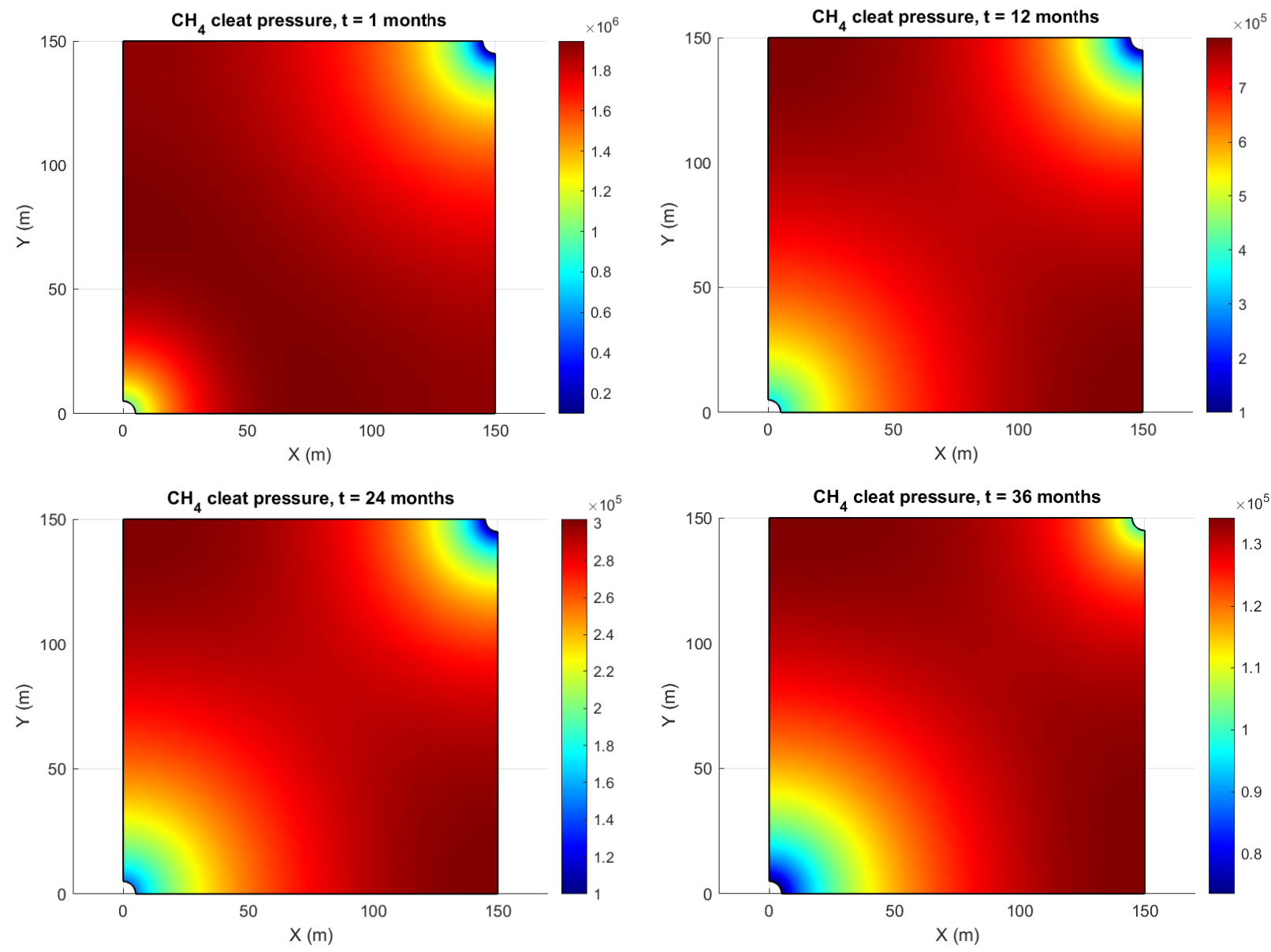

Figure 7: $\mathrm{CH}_{4}$ pressure profiles in the cleat for different times, $\mathrm{P}$ injection $=5 \mathrm{MPa}$ 

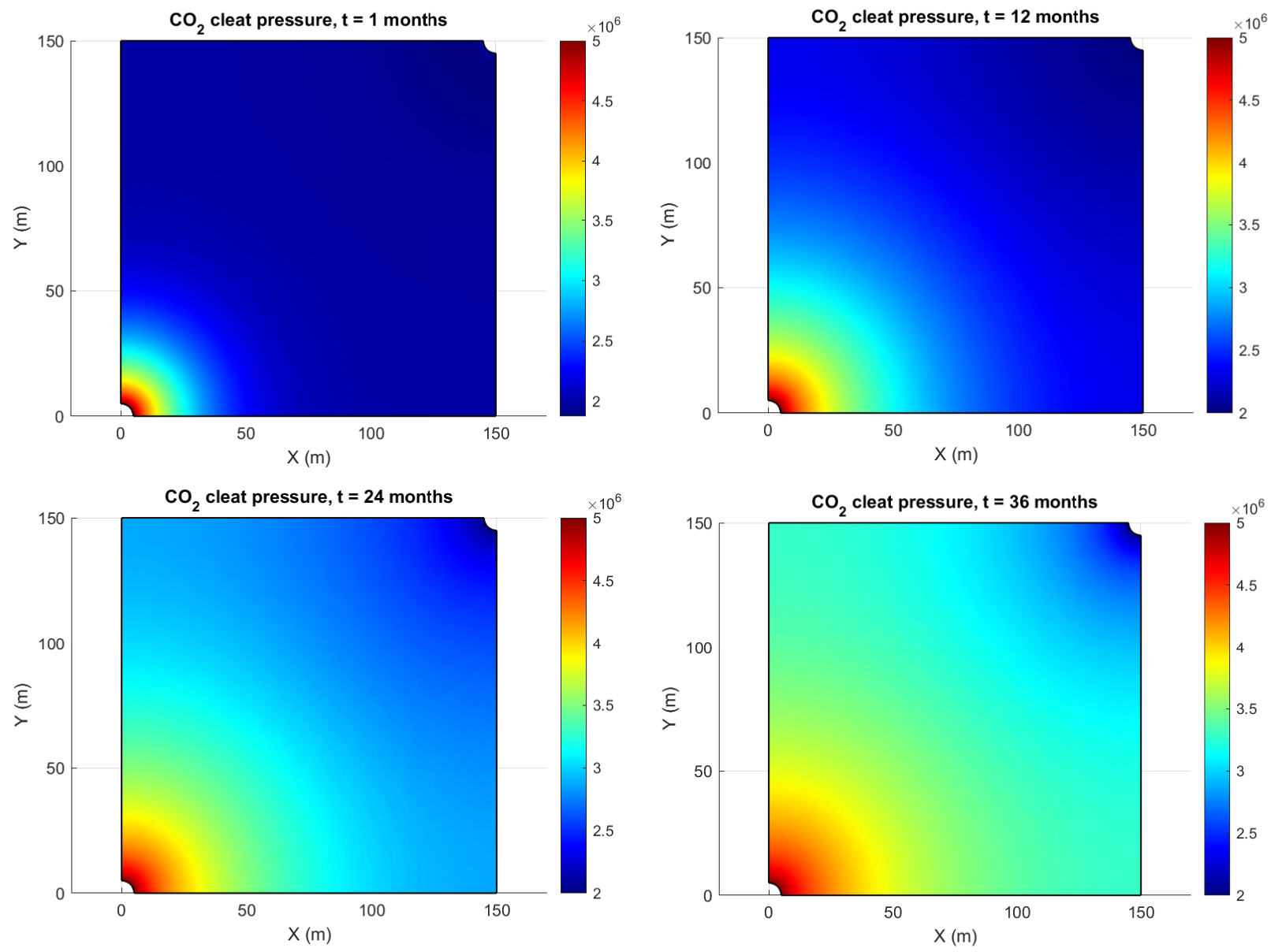

Figure 8: $\mathrm{CO}_{2}$ pressure profiles in the cleats for different times, $\mathrm{P}$ injection $=5 \mathrm{MPa}$ 

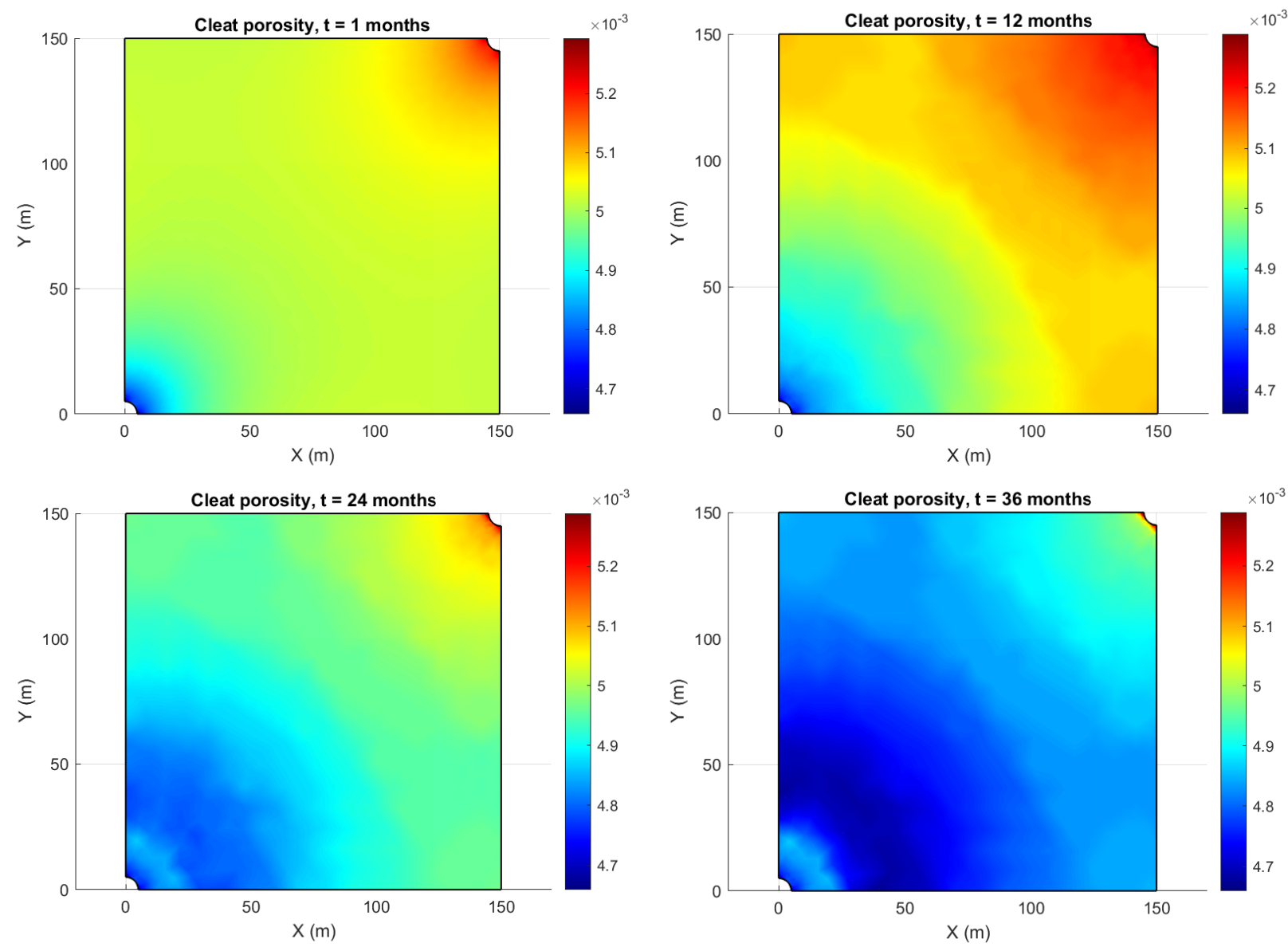

Figure 9: Cleat porosity for different times, $\mathrm{P}$ injection $=5 \mathrm{MPa}$ 

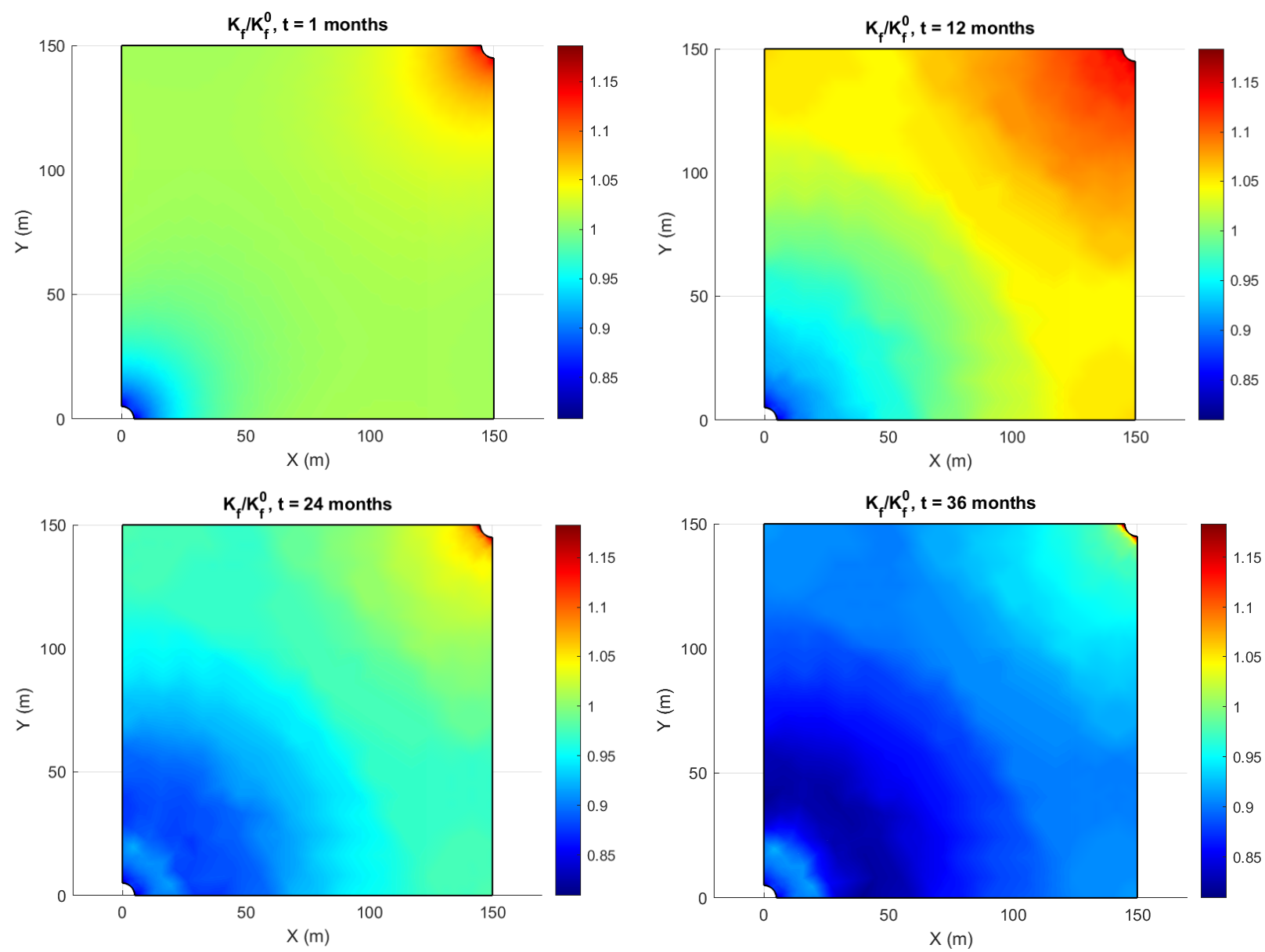

Figure 10: Ratio between the cleat permeability and its initial value for different times, $\mathrm{P}$ injection $=5 \mathrm{MPa}$

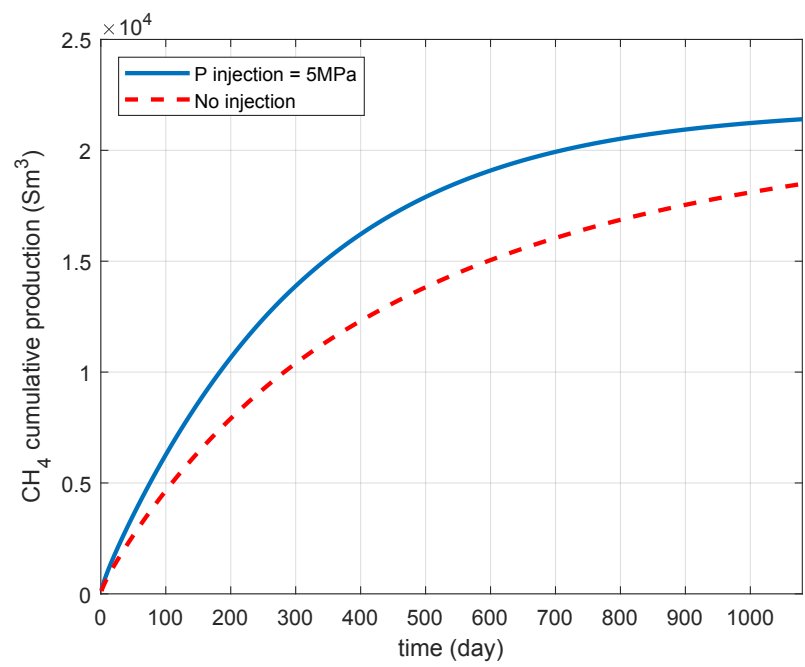

Figure 11: $\mathrm{CH}_{4}$ cumulative production in two scenarios: without injection and with $\mathrm{CO}_{2}$ injection, $\mathrm{P}$ injection $=5 \mathrm{MPa}$ 
[11] G. Wang, K. Wang, Y. Jiang, S. Wang, Reservoir permeability evolution during the process of $\mathrm{co}_{2}$ enhanced coalbed methane recovery, Energies 11 (2018) 1-21.

the counter-intuitive and experimentally observed cleats closure phenomena. Simulations showed that the cleat permeability decrease is significant near the injection well due to high injection pressure and can close the cleats if special attention is not paid to the injection pressure of $\mathrm{CO}_{2}$. The important result of this work is to illustrate the potential of the proposed multiscale approach to couple complex adsorption phenomena at the nanoscale with mechanics and transport problem at the reservoir scale.

\section{References}

[1] I. Gray, Reservoir engineering in coal seams: Part 1-the physical process of gas storage and movement in coal seams, SPE Reservoir Engineering 2 (1987) 28-34.

[2] J. Ayoub, L. Colson, J. Hinkel, D. Johnston, J. Levine, Learning to produce coalbed methane, Oilfield Review 3 (1991) 27-40.

[3] S. E. Laubach, R. A. Marrett, J. E. Olson, A. R. Scott, Characteristics and origins of coal cleat: A review, International Journal of Coal Geology 35 (1998) 175-207.

[4] J. Seidle, Fundamentals of coalbed methane reservoir engineering, PennWell Books, 2011.

[5] T. A. Moore, Coalbed methane: A review, International Journal of Coal Geology 101 (2012) 36-81.

[6] H. E. Ross, P. Hagin, M. D. Zoback, $\mathrm{Co}_{2}$ storage and enhanced coalbed methane recovery: Reservoir characterization and fluid flow simulations of the big george coal, powder river basin, wyoming, usa, International Journal of Greenhouse Gas Control 3 (2009) 773-786.

[7] C. O. Karacan, Swelling-induced volumetric strains internal to a stressed coal associated with $\mathrm{co}_{2}$ sorption, International Journal of Coal Geology 72 (2007) 209-220.

[8] T. D. Le, C. Moyne, M. A. Murad, I. Panfilov, A three-scale poromechanical model for swelling porous media incorporating solvation forces: Application to enhanced coalbed methane recovery, Mechanics of Materials 131 (2019) 47-60.

[9] J. Yi, I. Y. Akkutlu, C. O. Karacan, C. R. Clarkson, Gas sorption and transport in coals: A poroelastic medium approach, International Journal of Coal Geology 77 (2009) 137-144.

[10] Y. Wu, J. Liu, D. Elsworth, Z. Chen, L. Connell, Z. Pan, Dual poroelastic response of a coal seam to $\mathrm{CO}_{2}$ injection, International Journal of Greenhouse Gas Control 4 (2010) 668-678. 
[12] Y. Fan, C. Deng, X. Zhanga, F. Lid, X. Wanga, L. Qiao, Numerical study of $\mathrm{Co}_{2}$-enhanced coalbed methane recovery, International Journal of Greenhouse Gas Control 76 (2018) 12-23.

[13] B. Zhang, J. Zhu, D. Tang, Y. Jiang, The effect of adsorption-induced swelling on porosity based on the transient coal swelling model, AIP advances 09 (2019) 1-11.

[14] H. Kumar, D. Elsworth, J. P. Mathews, J. Liu, D. Pone, Effect of $\mathrm{co}_{2}$ injection on heterogeneously permeable coalbed reservoirs, Fuel 135 (2014) 509-521.

[15] S. Nikoosokhan, M. Vandamme, P. Dangla, A poromechanical model for coal seams saturated with binary mixtures of $\mathrm{ch}_{4}$ and $\mathrm{co}_{2}$, Journal of the Mechanics and Physics of Solids 71 (2014) 97-111.

[16] M. Vandamme, L. Brochard, B. Lecampion, O. Coussy, Adsorption and strain: The $\mathrm{co}_{2}$-induced swelling of coal, Journal of the Mechanics and Physics of Solids 58 (2010) 1489-1505.

[17] M. A. Murad, C. Moyne, A dual porosity model for ionic solute transport in expansive clays, Computational Geosciences 12 (2008) 47-82.

[18] T. D. Le, C. Moyne, M. A. Murad, A three-scale model for ionic solute transport in swelling clays incorporating ion-ion correlation effects, Advances in Water Resources 75 (2015) 31-52.

[19] T. D. Le, M. A. Murad, A. P. Pereira, A new matrix/fracture multiscale coupled model for flow in shale-gas reservoirs, SPE journal 22 (2017) 265-288.

[20] T. D. Le, M. A. Murad, A new matrix/fracture multiscale coupled model for flow in shale-gas reservoirs, Applied Mathematical Modelling 64 (2018) 453-479.

[21] E. Kierlik, M. L. Rosinberg, Density-functional theory for inhomogeneous fluids : Adsorption of binary mixtures, Physical Review A 44 (1991) 5025-5037.

[22] R. Roth, Fundamental measure theory for hard-sphere mixtures: a review, Journal of Physics: Condensed Matter 22 (2010) 63102-.

[23] Y. Rosenfeld, Free-energy model for the inhomogeneous hard-sphere fluid mixture and density functional theory of freezing, Physical Review Letters 63 (1989) 980-983.

[24] O. Coussy, Poromechanics, John Wiley \& Sons, 2004.

[25] J. L. Auriault, Heterogeneous media: Is an equivalent homogeneous description always possible?, Int. J. Engineering Science 29 (1991) 785-795.

[26] J. Lewandowska, A. Szymkiewicz, J. L. Auriault, Upscaling of richards's equation for soils containing highly conductive inclusions, Advances in Water Resources 28 (2005) 11591170. 
[27] T. Levy, Filtration in a porous fissured rock: influence of the fissures connexity, European Journal of Mechanics - B/Fluids 9 (1990) 309327.

[28] K. T. Lim, K. Aziz, Matric-fracture transfer shape factors for dual-porosity simulators, J. Pet. Sci. Eng. 13 (1995) 169-178.

[29] J. E. Warren, P. J. Root, The behavior of naturally fractured reservoirs, Society of Petroleum Engineers Journal 3 (1963) 245-255.

[30] H. Kazemi, L. S. Merrill, K. L. Portrfield, P. R. Zeman, Numerical simulation of water-oil flow in naturally fractured reservoirs, Society of Petroleum Engineers Journal 16 (1976) 317-326.

[31] Z. E. Heinemann, G. M. Mittermeir, Derivation of the kazemigilmanelsharkawy generalized dual porosity shape factor, Transport in Porous Media 91 (2012) 123132.

\section{Appendix A. Warren and Root approximation}

In this appendix, an approximation for the mass transfer term in the matrix in the sense of [29] is developed. In the isotropic case, the effective diffusion coefficient $\boldsymbol{D}_{e q, i}$ reduces to $D_{e q, i}$ so that from (23), the mass conservation equation for species $i$ in the matrix is given as

$$
\left\{\begin{aligned}
\frac{\partial\left(\phi_{m} G_{i} \rho_{m, i}\right)}{\partial t} & =\boldsymbol{\nabla} \cdot\left(D_{e q, i} \nabla \rho_{m, i}\right) & & \text { in } Y_{m} \\
\rho_{m, i} & =\rho_{f, i} & & \text { on } \partial Y_{f m} \\
\rho_{m, i} & =\rho_{m, i}^{i n i} & & \text { at } t=0
\end{aligned}\right.
$$

On the interface $\partial Y_{f m}$ between the cleats and the matrix, the mass density is imposed by the value in the cleats and an uniform initial condition is assumed. In addition, $\rho_{m, i}$ is a $Y$-periodic function.

The mass exchange term for species $i$ from the matrix to the cleats is given by

$$
Q_{m-f, i}=-\frac{1}{|Y|} \int_{\partial Y_{f m}} D_{e q, i} \boldsymbol{\nabla} \rho_{m, i} \cdot \boldsymbol{n}_{m f} \mathrm{~d} \Gamma
$$

where $\boldsymbol{n}_{m f}$ is the normal outside the matrix at the matrix/cleats interface. Using the periodic condition at the outer boundary $\partial Y_{m e}$ of the unit cell $Y$ and considering the divergence theorem and Eqs. (A.1), we have

$$
\begin{aligned}
Q_{m-f, i} & =-\frac{1}{|Y|} \int_{\partial Y_{f m} \bigcup \begin{array}{l}
D_{e q, i} \nabla \rho_{m e} \\
\rho_{m e}
\end{array}} \boldsymbol{n}_{m f} \mathrm{~d} \Gamma=-\frac{1}{|Y|} \int_{Y_{m}} \nabla \cdot\left(D_{e q, i} \boldsymbol{\nabla} \rho_{m, i}\right) \mathrm{d} V \\
& =-\frac{1}{|Y|} \int_{Y_{m}} \frac{\partial\left(\phi_{m} G_{i} \rho_{m, i}\right)}{\partial t} \mathrm{~d} V=-\frac{\partial}{\partial t}\left[\left(1-\bar{\phi}_{f}\right)\left\langle\phi_{m} G_{i} \rho_{m, i}\right\rangle^{m}\right]
\end{aligned}
$$

Neglecting the slow variation of $\phi_{m}$ and $G_{i}$ with time relative to the diffusion time, Eqs. (A.1) become

$$
\left\{\begin{aligned}
\phi_{m} G_{i} \frac{\partial \rho_{m, i}}{\partial t} & =\boldsymbol{\nabla} \cdot\left(D_{e q, i} \nabla \rho_{m, i}\right) & & \text { in } Y_{m} \\
\rho_{m, i} & =\rho_{f, i}(t) & & \text { on } \partial Y_{f m} \\
\rho_{m, i} & =\rho_{m, i}^{i n i} & & \text { at } t=0
\end{aligned}\right.
$$


The above problem can be solved using the following auxiliary problem

$$
\left\{\begin{aligned}
\frac{\partial U_{m, i}}{\partial t} & =D_{e q, i}^{*} \nabla^{2} U_{m, i} & & \text { in } Y_{m} \\
U_{m, i} & =1 & & \text { on } \partial Y_{f m} \\
U_{m, i} & =0 & & \text { at } t=0
\end{aligned}\right.
$$

where $D_{e q, i}^{*}=D_{e q, i} /\left(\phi_{m} G_{i}\right)$ is the apparent diffusion coefficient. The problem (A.5) is now written in the Laplace space in time with the Laplace variable $p$ and $\bar{U}_{m, i}=\mathcal{L}\left(U_{m, i}\right)$ designating the Laplace transform

355 of $U_{m, i}$. It yields

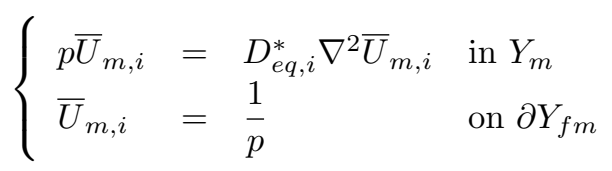

The solution of the problem (A.4) for $\bar{\rho}_{m, i}$ is easily obtained using the auxiliary problem (A.5). In the Laplace domain the exact solution is given by

$$
\bar{\rho}_{m, i}=p \bar{\rho}_{f, i} \bar{U}_{m, i}
$$

where $\bar{\rho}_{m, i}=\mathcal{L}\left(\rho_{m, i}-\rho_{m, i}^{i n i}\right)$ and $\bar{\rho}_{f, i}=\mathcal{L}\left(\rho_{f, i}-\rho_{m, i}^{i n i}\right)$. Averaging over the unit cell $Y$ leads to

$$
\left\langle\bar{\rho}_{m, i}\right\rangle^{m}=p \bar{\rho}_{f, i}\left\langle\bar{U}_{m, i}\right\rangle^{m}
$$

The exchange term (A.3) is given in the Laplace space by

$$
\bar{Q}_{m-f, i}=-\left(1-\bar{\phi}_{f}\right) \phi_{m} G_{i} p\left\langle\bar{\rho}_{m, i}\right\rangle^{m}=-\left(1-\bar{\phi}_{f}\right) \phi_{m} G_{i} p^{2} \bar{\rho}_{f, i}\left\langle\bar{U}_{m, i}\right\rangle^{m}
$$

360

By using relation (A.8),

$$
\left\langle\bar{\rho}_{m, i}\right\rangle^{m}-\bar{\rho}_{f, i}=\bar{\rho}_{f, i}\left(p\left\langle\bar{U}_{m, i}\right\rangle^{m}-1\right)
$$

the exchange term $\bar{Q}_{m-f, i}$ can be expressed in function of the gases density difference between the matrix and the cleats

$$
\begin{aligned}
\bar{Q}_{m-f, i} & =-\left(1-\bar{\phi}_{f}\right) \phi_{m} G_{i} p^{2} \frac{\left\langle\bar{U}_{m, i}\right\rangle^{m}}{p\left\langle\bar{U}_{m, i}\right\rangle^{m}-1}\left(\left\langle\bar{\rho}_{m, i}\right\rangle^{m}-\bar{\rho}_{f, i}\right) \\
& =p \bar{\alpha}_{i}\left(\left\langle\bar{\rho}_{m, i}\right\rangle^{m}-\bar{\rho}_{f, i}\right)
\end{aligned}
$$

where $p \bar{\alpha}_{i}=-\left(1-\bar{\phi}_{f}\right) \phi_{m} G_{i} p^{2} \frac{\left\langle\bar{U}_{m, i}\right\rangle^{m}}{p\left\langle\bar{U}_{m, i}\right\rangle^{m}-1}$. The inverse Laplace transform allows to write the exact solution of problem (A.4) in the form of a time convolution where $\alpha_{i}(t)=\mathcal{L}^{-1}\left(\bar{\alpha}_{i}(p)\right)$ :

$$
Q_{m-f, i}=\int_{\tau=0}^{t} \alpha_{i}(t-\tau) \frac{\partial}{\partial \tau}\left(\left\langle\rho_{m, i}\right\rangle^{m}-\rho_{f, i}\right)(\tau) \mathrm{d} \tau
$$

Rather than using the exact preceding expression, Warren and Root's approximation consists in replacing it with a simple product introducing the so-called matrix-fracture transfer shape factor $\alpha_{t, i}$ [29]:

$$
Q_{m-f, i}=\alpha_{t, i}\left(\left\langle\rho_{m, i}\right\rangle^{m}-\rho_{f, i}\right)
$$


There are several ways to determine this shape factor. One way is to compute the limit of the exact expression for large time values $\alpha_{t, i}=\lim _{p \rightarrow 0} p \bar{\alpha}_{i}$. If the matrix is a set of rectangular parallelepipeds of size $L_{x} \times L_{y} \times L_{z}, \alpha_{t, i}=12 D_{i}^{*}\left(1 / L_{x}^{2}+1 / L_{y}^{2}+1 / L_{z}^{2}\right)$. Other values are available in the literature [29, 28, 30,31].

\section{Appendix B. Upscaling of the nanoscopic model}

In this appendix, the homogenization procedure to upscale the nanoscopic model presented in the Section 3 is developed. From the scaling equations 14, we now collect the successive power of $\epsilon$.

\section{Slow variables}

The problem in $c_{b, i}^{(0)}$ periodic in $\mathbf{z}$ is written as

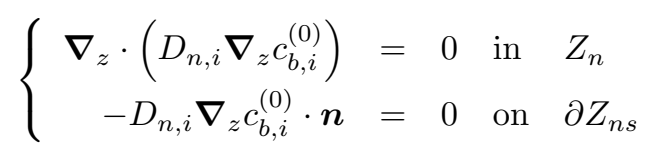

375

The problem in $c_{b, i}^{(1)}$ is given as

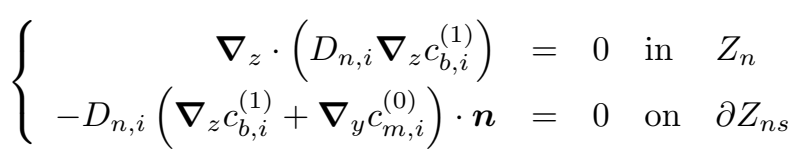

whose solution is

$$
c_{b, i}^{(1)}=\widehat{c}_{b}^{(1)}(t, \mathbf{y})-\mathbf{z} \cdot \nabla_{y} c_{m, i}^{(0)}
$$

where $\widehat{c}_{b}^{(1)}(t, \mathbf{y})$ plays the role of an integration constant. From Eq. B.4, we obtain immediately the flux in the nanopores at the order $\mathcal{O}\left(\epsilon^{-1}\right)$ given by

$$
\boldsymbol{j}_{n, i}^{(-1)}=-D_{n, i}\left(\boldsymbol{\nabla}_{z} c_{b, i}^{(1)}+\nabla_{y} c_{m, i}^{(0)}\right) \equiv 0
$$

385

Considering the Taylor development of $c_{b}(t, \mathbf{y}, \mathbf{z})$, one obtains

$$
\begin{aligned}
c_{b, i}(t, \mathbf{y}, \mathbf{z}) & =c_{m, i}^{(0)}(t, \mathbf{y})+\mathbf{z} \cdot \nabla_{y} c_{m, i}^{(0)}(t, \mathbf{y})+c_{b, i}^{(1)}(t, \mathbf{y}, \mathbf{z})+\mathcal{O}\left(\epsilon^{2}\right) \\
& =c_{m, i}^{(0)}(t, \mathbf{y})+\mathbf{z} \cdot \nabla_{y} c_{m, i}^{(0)}(t, \mathbf{y})+\widehat{c}_{b}^{(1)}(t, \mathbf{y})-\mathbf{z} \cdot \nabla_{y} c_{m, i}^{(0)}(t, \mathbf{y})+\mathcal{O}\left(\epsilon^{2}\right) \\
& =c_{m, i}^{(0)}(t, \mathbf{y})+\widehat{c}_{b}^{(1)}(t, \mathbf{y})+\mathcal{O}\left(\epsilon^{2}\right)
\end{aligned}
$$


We remark that in the unit cell $Z$, the concentration $c_{b, i}$ in the nanopores is constant at the order $\mathcal{O}\left(\epsilon^{2}\right)$.

The problem in $c_{s, i}^{(1)}$ is written in the solid matrix

$$
\left\{\begin{aligned}
\boldsymbol{\nabla}_{z} \cdot \boldsymbol{j}_{s, i}^{(0)} & =\boldsymbol{\nabla}_{z} \cdot\left[D_{s, i}\left(\boldsymbol{\nabla}_{z} c_{s, i}^{(1)}+\boldsymbol{\nabla}_{y} c_{m, i}^{(0)}\right)\right]=\boldsymbol{\nabla}_{z} \cdot\left(D_{s, i} \boldsymbol{\nabla}_{z} c_{s, i}^{(1)}\right)=0 & & \text { in } Z_{s} \\
c_{s, i}^{(1)} & =\widehat{c}_{b, i}^{(1)}(t, \mathbf{y})-\mathbf{z} \cdot \boldsymbol{\nabla}_{y} c_{m, i}^{(0)} & & \text { on } \partial Z_{n s}
\end{aligned}\right.
$$

with periodic boundary conditions on the external frontiers of the unit cell $\partial Z_{s e}$. The solution is searched in the form

$$
c_{s, i}^{(1)}(t, \mathbf{y}, \mathbf{z})=\widehat{c}_{b}^{(1)}(t, \mathbf{y})+\chi(\mathbf{z}) \cdot \nabla_{y} c_{m, i}^{(0)}(t, \mathbf{y})
$$

390

where the vector $\chi$ periodic in the unit cell is solution of

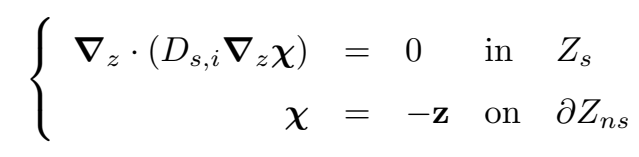

\section{Averaged equations}

Taking into account relation (B.5), the problem in $c_{b, i}^{(2)}$ is given as

$$
\left\{\begin{aligned}
\boldsymbol{\nabla}_{z} \cdot\left[D_{n, i}\left(\boldsymbol{\nabla}_{z} c_{b, i}^{(2)}+\nabla_{y} c_{b, i}^{(1)}\right)\right] & =0 & & \text { in } \quad Z_{n} \\
-D_{n, i}\left(\nabla_{z} c_{b, i}^{(2)}+\nabla_{y} c_{b, i}^{(1)}\right) \cdot \boldsymbol{n} & =-D_{s, i}\left(\boldsymbol{\nabla}_{z} c_{s, i}^{(1)}+\nabla_{y} c_{m, i}^{(0)}\right) \cdot \boldsymbol{n} & & \text { on } \quad \partial Z_{n s}
\end{aligned}\right.
$$

Finally the problems in $c_{b, i}^{(3)}$ and $c_{s, i}^{(2)}$ as well as the flux condition on the interface $\partial Z_{n s}$ are written as

$$
\left\{\begin{aligned}
\frac{\partial\left(G_{i} c_{m, i}^{(0)}\right)}{\partial t}= & \nabla_{z} \cdot\left[D_{n, i}\left(\nabla_{z} c_{b, i}^{(3)}+\nabla_{y} c_{b, i}^{(2)}\right)\right]+\nabla_{y} \cdot\left[D_{n, i}\left(\nabla_{z} c_{b, i}^{(2)}+\nabla_{y} c_{b, i}^{(1)}\right)\right] & \text { in } Z_{n} \\
0= & \nabla_{z} \cdot\left[D_{s, i}\left(\nabla_{z} c_{s, i}^{(2)}+\nabla_{y} c_{s, i}^{(1)}\right]+\nabla_{y} \cdot\left[D_{s, i}\left(\nabla_{z} c_{s, i}^{(1)}+\nabla_{y} c_{m, i}^{(0)}\right)\right]\right. & \text { in } Z_{s} \\
& -D_{n, i}\left(\nabla_{z} c_{b, i}^{(3)}+\nabla_{y} c_{b, i}^{(2)}\right) \cdot \boldsymbol{n}=-D_{s, i}\left(\nabla_{z} c_{s, i}^{(2)}+\nabla_{y} c_{s, i}^{(1)}\right) \cdot \boldsymbol{n} & \text { on } \partial Z_{n s}
\end{aligned}\right.
$$

Given the boundary condition on the interface $\partial Z_{n s}$ and periodicity conditions on the boundary of the cell 395 $\partial Z_{\text {se }}$, averaging the previous equations over the unit cell leads to

$$
\frac{\partial\left(\phi_{m} G_{i} c_{m, i}^{(0)}\right)}{\partial t}=-\frac{1}{|Z|} \nabla_{y} \cdot\left[\int_{Z_{n}} \boldsymbol{j}_{n, i}^{(0)} \mathrm{d} V+\int_{Z_{s}} \boldsymbol{j}_{s, i}^{(0)} \mathrm{d} V\right]=-\nabla_{y} \cdot\left\langle\boldsymbol{j}_{i}^{(0)}\right\rangle
$$

with

$$
\begin{aligned}
& \boldsymbol{j}_{s, i}^{(0)}=-D_{s, i}\left(\nabla_{z} c_{s, i}^{(1)}+\nabla_{y} c_{m, i}^{(0)}\right) \\
& \boldsymbol{j}_{n, i}^{(0)}=-D_{n, i}\left(\nabla_{z} c_{b, i}^{(2)}+\nabla_{y} c_{b, i}^{(1)}\right)
\end{aligned}
$$

where $\phi_{m}=\left|Z_{n}\right| /|Z|$ is the volume fraction of nanopores in the matrix. It should be noted that in the case of different pore diameters with different volumes $V_{k}$, we obtain

$$
G_{i}=\frac{\sum_{k}\left(G_{i}\right)_{k} V_{k}}{\sum_{k} V_{k}}
$$


Recall relations (B.7a) and (B.10)

$$
\boldsymbol{\nabla}_{z} \cdot \boldsymbol{j}_{s, i}^{(0)}=0 \text { in } Z_{s} ; \quad \boldsymbol{\nabla}_{z} \cdot \boldsymbol{j}_{n, i}^{(0)}=0 \text { in } Z_{n} ; \quad \boldsymbol{j}_{s, i}^{(0)} \cdot \boldsymbol{n}=\boldsymbol{j}_{n, i}^{(0)} \cdot \boldsymbol{n} \text { on } \partial Z_{n s}
$$

400

For a divergence-free flux $\boldsymbol{j}($ i.e. $\boldsymbol{\nabla} \cdot \boldsymbol{j}=0)$ in $Z_{*}$, we have

$$
\nabla \cdot(\boldsymbol{j} \otimes \mathbf{z})=\boldsymbol{j}+\mathbf{z} \nabla \cdot \boldsymbol{j}=\boldsymbol{j}
$$

Integrating the above relation in $Z_{*}$ leads to

$$
\int_{Z_{*}} \boldsymbol{j} \mathrm{d} V=\int_{Z_{*}} \boldsymbol{\nabla} \cdot(\boldsymbol{j} \otimes \mathbf{z}) \mathrm{d} V=\int_{\partial Z_{*}} \boldsymbol{n} \cdot(\boldsymbol{j} \otimes \mathbf{z}) \mathrm{d} S=\int_{\partial Z_{*}} \mathbf{z}(\boldsymbol{j} \cdot \boldsymbol{n}) \mathrm{d} S
$$

where $\boldsymbol{n}$ is the outward normal to $Z_{*}$ on the boundary $\partial Z_{*}$ of the domain.

Applying this relation to the two fluxes $\boldsymbol{j}_{n, i}^{(0)}$ and $\boldsymbol{j}_{n, i}^{(0)}$ gives

$$
\begin{aligned}
\int_{Z_{n}} \boldsymbol{j}_{n, i}^{(0)} \mathrm{d} V & =\int_{\partial Z_{n s}} \mathbf{z}\left(\boldsymbol{j}_{n, i}^{(0)} \cdot \boldsymbol{n}_{n}\right) \mathrm{d} S \\
\int_{Z_{s}} \boldsymbol{j}_{s, i}^{(0)} \mathrm{d} V & =\int_{\partial Z_{n s}} \mathbf{z}\left(\boldsymbol{j}_{s, i}^{(0)} \cdot \boldsymbol{n}_{s}\right) \mathrm{d} S+\int_{\partial Z_{s e}} \mathbf{z}\left(\boldsymbol{j}_{s, i}^{(0)} \cdot \boldsymbol{n}_{s}\right) \mathrm{d} S
\end{aligned}
$$

Adding both relations with $\boldsymbol{n}_{n}=-\boldsymbol{n}_{s}$ on $\partial Z_{n s}$ taking into account the closure relation for $c_{s, i}^{(1)}$ leads to the 405 average total flux

$$
\begin{aligned}
\left\langle\boldsymbol{j}_{i}^{(0)}\right\rangle & =\frac{1}{|Z|} \int_{\partial Z_{s e}} \mathbf{z}\left(\boldsymbol{j}_{s, i}^{(0)} \cdot \boldsymbol{n}_{s}\right) \mathrm{d} S=-\frac{1}{|Z|} \int_{\partial Z_{s e}} \mathbf{z} D_{s, i}\left[\left(\boldsymbol{\nabla}_{z} c_{s, i}^{(1)}+\boldsymbol{\nabla}_{y} c_{m, i}^{(0)}\right) \cdot \boldsymbol{n}_{s}\right] \mathrm{d} S \\
& =-\frac{1}{|Z|}\left[\int_{\partial Z_{s e}} D_{s, i}\left(\mathbf{z} \otimes \boldsymbol{n}_{s}\right) \cdot\left(\boldsymbol{I}+\boldsymbol{\nabla}_{z} \boldsymbol{\chi}\right) \mathrm{d} S\right] \cdot \boldsymbol{\nabla}_{y} c_{m, i}^{(0)}=-\boldsymbol{D}_{e q, i} \cdot \boldsymbol{\nabla}_{y} c_{m, i}^{(0)}
\end{aligned}
$$

with the effective diffusion coefficient defined as

$$
\boldsymbol{D}_{e q, i}=\frac{1}{|Z|}\left[\int_{\partial Z_{s e}} D_{s, i}\left(\mathbf{z} \otimes \boldsymbol{n}_{s}\right) \cdot\left(\boldsymbol{I}+\boldsymbol{\nabla}_{z} \boldsymbol{\chi}\right) \mathrm{d} S\right]
$$

If the unit cell is a rectangular parallelepiped $\left(\ell_{1} \times \ell_{2} \times \ell_{3}\right)$ with $\Sigma_{j}$ designating the facet relative to the normal $\boldsymbol{n}_{j}$, by using periodic condition, the averaged diffusion coefficient is given by

$$
\left(\boldsymbol{D}_{e q, i}\right)_{j k}=\frac{1}{\left|\Sigma_{j}\right|}\left[\int_{\Sigma_{j}} D_{s, i}\left(\delta_{j k}+\frac{\partial \chi_{k}}{\partial z_{j}}\right) \mathrm{d} S\right]
$$

The averaged transport equation in the matrix (B.12) can be obtained

$$
\frac{\partial\left(\phi_{m} G_{i} c_{m, i}^{(0)}\right)}{\partial t}=\nabla_{y} \cdot\left(\boldsymbol{D}_{e q, i} \cdot \nabla_{y} c_{m, i}^{(0)}\right)
$$

